\title{
Transportation network design for maximizing space-time accessibility
}

\author{
Lu Tong $^{\mathrm{a}}$ \\ Email: 1tong@bjtu.edu.cn \\ Xuesong Zhou ${ }^{\mathrm{b}, *}$ \\ Email:xzhou74@asu.edu \\ (Corresponding Author) \\ Harvey J. Miller ${ }^{\mathrm{c}}$ \\ Email: miller.81@osu.edu

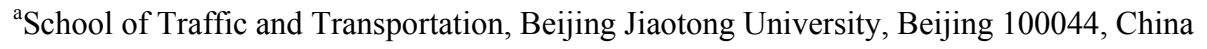 \\ ${ }^{\mathrm{b}}$ School of Sustainable Engineering and the Built Environment, Arizona State University, Tempe, AZ \\ 85287, USA \\ ${ }^{\mathrm{c}}$ Department of Geography, The Ohio State University, Columbus, OH 43210, USA \\ *Corresponding author.Tel.: +1 4809655827
}

\begin{abstract}
One of the goals of transportation system construction and management is to improve individuals' accessibilityor the ease of reaching desired activities, destinations and services. However, manytransportation network design models instead focus on maximizing individuals' mobilityor the ease of movement within the network. By adapting a space-time prism analysis framework, this paper aims to address a new urban network design problemto maximizethe system-wide transportation accessibility between major activity locations, subject to a given highway construction budget. By constructing a time-dependent space-time network, we formulate the problem as a linear integer programming model to maximizethe number of accessible activity locations within travel time budget for road users. A Lagrangian relaxation solution framework effectively decomposes the original complex problem into classical subproblems such as knapsack and time-dependent least cost problems. Various examples and discussions are provided to consider the effectiveness of the proposed method in modeling accessibility-enhancement strategies such as congestion mitigation and land use policies.
\end{abstract}

Keywords: Accessibility; Space-time network;Network design;Lagrangian relaxation 


\section{Introduction}

As an essential performance measure for transportation systems, accessibility refers to the ability for travelers and freight shippers to reach desired activities, destinations and services. This contrasts with mobility, or the ease of movement within the network.Traditional transportation planning methods, including network design methods, focus on improving mobility-based measures such as speed and travel times. However, a mobility-oriented approach to transportation planning can induce travel demand and lead to traffic congestion (Handy, 2005). Although planning for mobility can improve network accessibility, accessibility-based planning expands the range of potential solutions compared to mobility-oriented strategies (Litman, 2003). Urban transportation planning for accessibility is a challenge for the metropolitan planning organizations (MPOs). One obstacle is a lack of methods for addressing accessibility directly rather than mobility in transportation network design (Santoset al., 2008).

In this paper, we formulate and solve the transportation network design problem with a focus on maximizing temporal and spatial accessibility. We introduce a new class of network design problem for maximizing the accessibility given travel time budget (TTB) constraints. By constructing a time-expanded network to model the space-time prism, a sensitive measure of individual accessibility (Hägerstrand, 1970), we develop a network design model with time-dependent arcs, while the equivalent optimization goal is to minimize individual inaccessibility between major activity locations in the space-time network. To address the computational efficiency issue for real-world large-scale applications, we develop a Lagrangian relaxation algorithm for effective problem decomposition.

The remainder of this paper proceeds as follows. The next section provides background on accessibility measures and network design for accessibility. In Section 3, a conceptual illustration is presented to describe the definition and calculation procedure of network accessibility under space-time network structure. Section 4 develops a linear integer programming model for the network design problem that aims to maximize system accessibility. Section 5 describes the Lagrangian relaxation solution framework and Section 6 provides discussions on various issues related to applying the accessibility-oriented network design model in planning practices.In Section 7, several demonstrative examples are presented to model various accessibility-enhancement strategies. The final section concludes the paper with some brief remarks.

\section{Background}

\subsection{Accessibility measures}

In transportation planning, accessibility is first defined by Hansen (1959) as the potential of opportunities for traveler interaction. Typically, accessibility captures the extent of attractiveness of each potential destination and some researchers represent accessibility as amount of activity potential reachable within a given travel time from an origin location (Chen et al., 2011).Most existing measures of accessibility can be further classified into the following categories.

i. Cumulative opportunity measurescount the number of potential opportunities that can be reached by each location within a given travel distance or travel time; locations with larger number of opportunities have higher accessibility (Wachs and Kumagai, 1973; Vickerman, 1974;El-Geneidyand Levinson, 2006).

ii. Spatial interaction-based measures balance the attractiveness of each opportunity against it travel cost from a given location 1959;Wilson,1971;Williams,1976;Erlander,1977;Weibull,1976, 1980;Geertmanand Ritsema 
Van Eck, 1995). Benefit measures use the economic concepts of utility theory and consumer surplus to measure accessibility (Ben-Akiva and Lermand, 1979, 1985;Neuburger, 1971;Small and Rosen 1981; Williams 1976, 1977).

iii. People-based accessibility measuresare sensitive to social, economic, cultural and demographic differences in accessibility imposed by differences in scheduling and location constraints as well as transportation resources. The space-time prism (Hägerstrand 1970) is a central concept,and modelers can either directly or indirectly incorporate prism-based space-time constraints into other measures such as spatial interaction or benefit measures (Miller 1991, 1999).

Many studies use accessibility measures in different transportation applications. Daganzo (2010) analyzes the structure of urban transit network for increasing accessibility. Hou and Li (2011) and Chen et al. (2014) study the impact of transport infrastructure development; Sohn (2006), Chen et al. (2007) and Taylor (2008) perform transportation network vulnerability analyses under network disruption scenarios, due to traffic incidents or flood damage. In these studies, the accessibility measure is typically used to represent the node-to-node connectivity, and researchers have been focusing on the evaluation or analysis of different strategies.

Many MPOs are seeking for viable models and approaches that can better design and improve the transportation networks to provide comprehensive services/utilities to general demand in an urban setting (Waddell 2002;Litman 2003, 2011; Curtis and Scheurer, 2010). The accessibility perspective has been widely adopted in the transportation planning field. It is used as a collective indicator for evaluating the overall transportation system performance (e.g., El-Geneidy and Levinson, 2006; Handy, 2005;Litman, 2011) and representing the general traveling opportunities. Other topics of interest for accessibility planning is how to jointly improve the sustainability and accessibility of cities through public transportation services, that is, offer feasible transit routes from major origins to desirable destinations within the available time frame. In our proposed research, weparticularly consider the planning or optimization aspect of the network design process, with a special focus on the integration of space-time accessibility measuresin general activity-travel decisions.

\subsection{Network design and accessibility}

In the field of operations research, the network design problem was first formulated by Dantzig(1965) as a fixed charge transshipment problem, and this class of problems has been well studied with a full spectrum of strategic, tactical, and operational decision-making situations. When it comes to transportation planning, the network design problem aims to find an optimal allocation and utilization of resources to achieve a certain goal (Crainic, 2000), such as improving traveler mobility, reducing air/noise pollution, avoiding accident and increasing accessibility.Magnantiand Wong (1984) review various integer programming-based network design models; a more up-to-date survey by Crainic (2000) focuses onthe classification and formulations of various service network design models for freight transportation. For the design and scheduling of transit network, Guihaireand Hao(2008) present a unified review of the crucial strategic and tactical steps of transit planning.A recent comprehensive survey on the urban transportation network design problem by Farahaniet al. (2013) specifically highlights the needs for bridging the modeling gaps between the road network design problem and public transit network design research. 
In general, common optimization criteriain transportation network design include road/traveler mobility, overall system reliability, emission measures at varying levels of spatialaggregation. The decision variables aretypically discrete in nature (as also shown by Miandoabchi et al., 2013; Wang et al., 2013), the design problems are often cast in various forms of integer or mixed integer programming models with NP-hard complexity.Many integer programming solution approaches have been developed along this research line. As an example, Ortega and Wolsey (2003) provide a branch-and-cut algorithm to solve the single-commodity, uncapacitated, fixed-charge network design problem.

There are also a number of previous studies using various accessibility measures in network design models, but they have focused on specific applications. In comparison, our proposed research aims to present a general and mathematically tractable model for considering travel time budgets and accessibility. The study by Viswanath and Peeta (2003)aims to minimize travel time and maximize accessibility at each demand center, but their accessibility measure is computed by determining if an Origin-Destination (OD) pair is still connected after an earthquake event. Murawski and Church (2009) measure accessibility based on whether each demand center is covered within a pre-specified distance from hospitals. Santos et al. (2008) describe a transportation network design problem based on equity and accessibility measures, and their accessibility measure is defined as a non-linear generalized cost function of link selection variables. In comparison, our model represents the accessibility through a virtual arc construct in a space-time network with linear structure, which can be solved through efficient space-time shortest path algorithms.

Recent studies onthe transportation network design problem start concentrating ona wide range of practically useful constraints and realistic scenarios. Ukkusuri et al.(2007) develop a robust traffic network design problem under uncertain origin-destination demand volume, solved through Genetic Algorithm-based heuristics. To improve the solution quality ofthe network design problem, Aguado (2009) propose a Lagrangian relaxation-based heuristic solution approach to solve the fixed charge transportation network design problem. Kennington and Nicholson (2010) introduce a time-space concept into the logistics network design problem and modeled it as a mixed binary linear program. Luathep et al. (2011) examine the transportation network design problem as a mathematical programming with equilibrium constraints and developed a global optimization algorithm based on a cutting constraint method.An activity-based network design problem is proposed by Kang et al. (2013), where the network design problem and the disaggregate household itinerary optimization problem are incorporated in a bi-level mathematical model. Jiang and Szeto (2014) develop a bi-level time-dependent discrete road network design problem considering integrated travelers and residents health impacts.

Table 1 provides a systematic comparison of key modeling components in the existing network design research. To better highlight the characteristics of our proposed network design model focusing on accessibility maximization, we would like to offer the following remarks. Most studies either assume deterministic traffic demand volume from origin to destination zones, or model uncertain traffic demand through constructing a deterministic equivalent based on known probability distributions. Accordingly, a majority of studies aim to minimize the total expected travel costs for all road users, with a consideration of system design costs as constraints or secondary objective functions. In comparison, rather than addressing travel demand, our model addresses a higher level of decisions pertaining to a general concept measure of space-time accessibility between major activity locations. In this case, we do not require the information on the actual number of demand volume as the 
accessibility measure is commonly used as a strategic indicator for current and future traveling opportunities.

Table 1 provides a systematic comparison of key modeling components in the existing network design research. To better highlight the characteristics of our proposed network design model focusing on accessibility maximization, we would like to offer the following remarks. Most studies either assume deterministic traffic demand volume from origin to destination zones, or model uncertain traffic demand through constructing a deterministic equivalent based on known probability distributions. Accordingly, a majority of studies aim to minimize the total expected travel costs for all road users, with a consideration of system design costs as constraints or secondary objective functions. In comparison, rather than addressing travel demand, our model addresses a higher level of decisions pertaining to a general concept measure of space-time accessibility between major activity locations. In this case, we do not require the information on the actual number of demand volume as the accessibility measure is commonly used as a strategic indicator for current and future traveling opportunities.

\section{(Insert Table 1)}

\section{Conceptual model}

This section illustrateshow to use the space-time network and related traveler activity trajectoriesto precisely represent network accessibility under TTB constraints.

\subsection{Space-time network}

Widely used in both transportation geography and transportation network modeling literature, the concept of space-time networksaims to integratephysical transportation networks with travelers' time-dependent movements/trajectories. To constructaspace-time network, the analysis time horizon is first divided into a series of intervals with the same time length $\sigma$. In Fig. 1,a physical transportation networkis shown on the lower portion, while the upper part plots a set of vertexes, traveling arcs and waiting arcs, eachwithdifferent spatial and temporal characteristics. Specifically, aperson, traveling in thisillustrative three-node network, departs from origin node $o$ at time $t_{0}+\sigma$ andarrives at node $a_{1}$ at time $t_{0}+2 \sigma$. The traveler finally reaches at the third node $a_{2}$ at time $t_{0}+6 \sigma$. The route choice decision can be described as a sequence of arcs traveling at this space-time network, namely the space-time travelingarcs $\left(o, a_{1}, t_{0}+\sigma, t_{0}+2 \sigma\right),\left(a_{1}, a_{1}, t_{0}+2 \sigma, t_{0}+3 \sigma\right)$ and $\left(a_{1}, a_{2}, t_{0}+3 \sigma, t_{0}+\right.$ $6 \sigma)$.The space-time network in Fig. 1 (typically used in the literature of transportation network modeling) corresponds to the illustration in Fig. 2, where these arcs tracing the movement of an individual form aspace-time path(Hägerstrand 1970). It should be noted that travel time between node $a_{1}$ and $a_{2}$ varies according to road congestion. If a traveler departs from node $a_{1} \sigma$ time earlier, say at time $t_{0}$, he/she can arrive at node $a_{2} 2 \sigma$ time earlier.

(Insert Fig. 1)

(Insert Fig. 2) 


\subsection{Space-time network accessibility}

Using Fig. 3, we now further characterize accessibility measures withtraveling timebudget (TTB) constraints.An activity location is defined as accessible from a particular location if it can be reached within time budget $T \sigma$ using the maximum or typical traveling speed. In this example, it is easy to verify that, the earliest possible time arriving at node $a_{1}$ and node $a_{2}$ are $t_{0}+2 \sigma$ and $t_{0}+5 \sigma$, respectively. If TTB is set as $T \sigma=4 \sigma$, then node $a_{1}$ is accessible while node $a_{2}$ is inaccessible for travelers originating at node $o$.

\section{(Insert Fig. 3)}

We now proceed to the more general case wherea road user departs from his/her origin location (e.g. home), arrives at an intermediate destination for the entire trip (e.g. workplace, shopping mall, hospital), performs an activity at that location, and finally returns to the origin location.To capture connected trips in a complete traveling tour,Fig. 4 illustrates the traveling sequence of a traveler withan activity performing time of $2 \sigma$ and aTTBof $7 \sigma$. One can easily verify that, activity location $a_{1}$ is accessible in this case for the given TTB constraint, whileactivity location node $a_{2}$ does not allowthe completion of the entire tour so it can be classified as inaccessible. To ensure the flowbalance requirement for the network flow model used in the following sections, we assume an individual stays at the origin node after he/she finishes the final leg of a trip. This corresponds to a waiting arc $\left(o, o, t_{0}+6 \sigma, t_{0}+7 \sigma\right)$ from the arrival time of $6 \sigma$ to the TTB of $7 \sigma$ in Fig. 4.

\section{(Insert Fig. 4)}

An important conceptfor analyzingtravelers' accessibility isspace-time prism (STP); this is the envelope of all possible space-time paths between two locations and times. Fig. 5 illustrates a simpleSTP in planar space with no activity time at an intermediate location. The spatial and temporal regionbounded by the prism is also called the potential path space (PPS), which measures the ability to reach (be coincident with) locations in space and time, given the locations and durations of fixed activities.Projecting PPS to the two-dimensional geographical planedelimits the potential path area (PPA); this is the set of geographical locations that a person can occupy (Wu and Miller, 2001; Miller, 2005).

\section{(Insert Fig. 5)}

To better connect the transportation planning/land use strategies with accessibility-enhancement goals, Fig. 6 shows a STP with stationary activity time (the cylinder connecting the two cones, representing when the individual is stationary) and highlights accessible/inaccessible activity locations. In this example of daily commuting, commuters have a total endurable time budget $T$ for both travel and work tasks. If commuters need to work for $T_{w}$ hours at their working locations, then this leads to a remaining time of $T-T_{w}$ for themto travel between a pair ofthe work and home locations.

(Insert Fig. 6) 
In order to account for possible congestion effect caused by time-varying network flow, we extend the classical STP concept to dynamic space-time prism(DSTP) framework, which adopts time-dependent travel time within a transportation network. Similarly, a dynamic potential path area (DPPA) can be found given a specified departure time.

Under the DPPA framework, the accessibility measure can be further defined in the following compact form (Wu and Miller, 2001). Given the overall time budget $T(o, a)$ and departure time $\tau$, the dynamic opportunity set $M_{o}$ of valued activity locations from location $o$ is,

$M_{o}=\left\{a \in \Omega \mid t^{\tau}(o, a)+t_{a}^{m}+t^{\tau^{\prime}}(a, o) \leq T(o, a)\right\}$.

That is, a location a is accessible if the total time budget is greater than the sum of travel time $t^{\tau}(o, a)+t^{\tau^{\prime}}(a, o)$ and minimum required participation time $t_{a}^{m}$, where the departure time from the activity location $\tau^{\prime}=\tau+t^{\tau}(o, a)+t_{a}^{m}$. Therefore, in the proposed space-time modeling structure, the congestion effect can be reflected by explicitly incorporating time-dependent link travel time into the accessibility measure for different departure times. As illustrated in Fig. 6, the travel speed before the traveling to the activity location (say in the morning) is significantly different from the speed after performing the activity (say in the evening), as a result of congestion.

In our model, we maximize the number of accessible activity locations(nodes) within the DSTP framework, which is equivalent to minimizing the number of inaccessible activity locations. It should be also remarked that, the general concept of transportation accessibility can be affected by a wide range of factors such as location attributes, road toll, comfort and risk. Without a loss of generality, our proposed network design model focuses on the major factor of travel time.

\section{Network design model}

We now describe the formal problem statement for the network design model for maximizing accessibility. First, the transportation network under consideration consists of a finite set of nodes $N$, and a finite set of directed transportation links $E$ between different adjacent nodes. We denote origin node $o$ andactivitynode $a$, while activity locations are a set of nodeso, $a \in P$ and $P \in N$. Index $t_{0}$ specifies the given departure time from the origin node, $T(o, a)$ denotesthe given time budget for origin-activity pair $(o, a)$.Tables 2 and 3 list the notations for the key parameters and variables.

\subsection{Notations}

(Inset Table 2)

(Insert Table 3)

\subsection{Building the space-time network}

Given node set $N$ (with a set of activity locations $P$ ) and link set $E$, the next task is to build the space-time network structure that can model the network-based PPS and identify the accessible locations in thecorresponding PPA. In the space-time networkto be built, we need to consider the following requirements: (1) ensure TTB constraint, (2) perform activity for a duration of $T^{a}$ time units, and (3) connect the space-time arcs with the physical links to be constructed.

\section{Algorithm 1: Build a space-time network for origin-activity pair $(o, a)$ departing at time $\tau$} Step1: Build space-time vertex set $Q$

Add vertex $(i, t)$ to $Q$ for $i \in N$ and each $t$. 


\section{Step 2: Build space-time arc set $A$}

Step 2.1: Add space-time traveling $\operatorname{arc}(i, t),(j, t+T T(i, j, t))$ to $A$, for link $(i, j) \in E$, where $T T(i, j, t)$ is the link travel time from node $i$ to node $j$ starting at time $t$.

Step 2.2: Adda set of space-time waiting arcsfor a pair of vertexes $(o, t),(o, t+1)$ to $A$, for each time $t$.

Step 2.3: Add a set of space-time activity-performing $\operatorname{arcsfor}$ a pair of vertexes $(a, t),(a, t+$ $\left.T^{a}\right)$ to $A$, for each time $t$.

Step 2.4: Add a space-time virtual traveling arc for a pair of vertexes $(o, \tau),(o, \tau+T(o, a))$ to $A$.

Step 3: Set arc cost structure and activity-performing coefficients

Step 3.1: Set $c_{i, j, t, s}(o, a, \tau)=1$ for virtual traveling $\operatorname{arcs}(i, j, t, s) \in A^{V}$

Step 3.2: Set $g_{i, j, t, s}(o, a, \tau)=1$ for space-time arcs $(i, j, t, s)$ in both sets $A^{A}$ and $A^{V}$

We illustrate the concept of space-time network building and accessibility maximization using the hypothetical 3-node network with given (time-invariant) link travel times in Table 4. Fig. 7 provides a visual representation.

\section{(Insert Table 4)}

(Inset Fig. 7)

Fig. 7 shows two cases: (a) without link $\left(o, a_{2}\right)$ and (b) with link $\left(o, a_{2}\right)$. First,we can construct the space-time traveling arcs only when their corresponding physical links exist.

To construct an equivalent network flow model with essential flow balance conditions at each vertex, we need to add virtual traveling arcs connecting the same origin node but different time stamps $t_{0}$ and $t_{0}+T$, that is, connect the super source vertex with the super sink vertex from the beginning time of 7:00 to the end time of 17:00. After further introducing i) waiting arcs at the origin, and ii) activity-performing arcs at the activity location, we now can illustrate how a feasible tour is generated within the space-time prism (with both total time budget constraint and activity-performing constraint). It is important to notice that, in each plot in Fig. 7, there are in fact two overlapping space-time networks for the same origin node $o$, one for activity location $a_{1}$ and the other for activity location $a_{2}$. Thus, for each activity location $a_{1}$ or $a_{2}$, we can identify the corresponding activity-performing arcs and virtual traveling arcs. That is, two red dashed lines represent virtual arcs for the origin-activity location pair with its activity location at $a_{1}$ and $a_{2}$, respectively. One can observe that the slope of the arc from the origin to node $a_{2}$ in Fig. 7(b) is slightly different from that in the Fig. 7(a). In our example, we specifically show this subtle difference to recognize a direct physical link $\left(o, a_{2}\right)$ is added in Fig. 7(b). Before this link is built (in Fig. 7(a)), if a traveler wants to go to location $a_{2}$, he/she must visit location $a_{1}$ first, and the travel time is $2+3=5$ hours. While under the scenario of Fig. $7(\mathrm{~b})$, travelers can reach location $a_{2}$ directly without going to location $a_{1}$, and the travel time reduces to 4 hours. Therefore, the slopes, which can be considered as travel speed or inverse of travel time, are not the same in these two subfigures.

Let us consider a person living at node $o$ with a 10-hour budget from 7:00 to 17:00 to travel. He or she plans to participate in a two-hour activities (e.g. shopping or part-time work) located at one of two nodes $a_{1}$ and $a_{2}$, and then travel back home. For the network design scenario shown in Fig. 7 (a), we can find a feasible tour from node $o$ to node $a_{1}$, then back to node $o$, while satisfying both time 
budget and activity-performing constraints. Thus, we declare node $a_{1}$ as accessible. However, node $a_{2}$ is not accessible through the physical network within the total time budget, so the virtual traveling arc that corresponds to $a_{2}$ will be selected to ensure the general flow balance constraint.On the other hand, if a link $\left(o, a_{2}\right)$ is built,as shown in Fig. 7 (b), thenthe person of interest will be able to travel from $o$ to both nodes directly and node $a_{2}$ now becomes accessible. Virtual arcs indicate the inaccessible level of certain origin-activity pairs within a travel time budget constraint. That is, if the resulting travel time is greater than the time budget, then the virtual arc is automatically selected as a result of cost minimization and flow balance constraint.

The above example focuses on a specificorigin-activity-origin (OAO) pair, or equivalent to the commonly used OD (origin-to-intermediate destination) pair. The next stage is how to quantify the overall accessibility for all origin-activity pairs (that is, for all activity locations from all origins). To do so, we first need to construct individual space-time networksby repeating Algorithm 1 for each origin-activity location pair. That is, if there are $|P|$ activity nodes, then we need to construct a total of $|P| \times(|P|-1)$ space-time networks. Each space-time network corresponding to the origin-activity location pair $(o, a)$ has one single virtualarc $(o, o, \tau, \tau+T(o, a))$, where $\tau$ is the departure time at orgin $o$. To comprehensively represent the system-wide accessibility through multiple copies of space-time networks, we use a 6-dimensional vector for space-time arcs, leading to a 7-dimensional binary variable $x_{i, j, s, t}(o, a, \tau)$.

It should be remarked that, the return trip consideration in our proposed accessibility measure is consistent with the classic time geography literature for space-time prism (e.g., Miller, 1991). On the other hand, one can also consider different combinations of trips in measuring accessibility, for example, origin to destination, origin to activity location then return back to the origin (as illustrated in Fig. 4), or a more complex case that involves a number of activities though a trip chaining construct (e.g., Bowman et al., 2001;Recker, 2001; Dong et al., 2006; Kang et al., 2013).

\subsection{Time-dependent network flow programming model}

Problem statement.Given the total construction budget constraint, the network design problem for maximizing system-wide transportation accessibility aims to find a set of transportation links to be addedto maximize the number of potential accessible destination nodes within individuals' time budget constraints. This problem will be reformulated to an equivalent time-dependent network design optimization problem with special cost structures.

Space-time flow balance constraints. To depict a time-dependent tour in the space-time network, we formulate a set of flow balance constraintsas follows.

$$
\sum_{(j, s) \in Q:(i, j, t, s) \in A} x_{i, j, t, s}(o, a, \tau)-\sum_{(j, s) \in Q:(j, i, s, t) \in A} x_{j, i, s, t}(o, a, \tau)=\left\{\begin{array}{rr}
1, & i=o, t=\tau \\
-1, & i=o, t=\tau+T(o, a), \\
0, & \text { otherwise }
\end{array}\right.
$$

for all $o, a \in P, \tau \in H(2)$

Thesespace-time flow balance constraintscan be denoted by an abbreviated form of $B_{o, a, \tau} \hat{x}(o, a, \tau)=$ $b_{o, a, \tau}$. For example, as shown in Fig. 7 (a), the flow corresponding to inaccessible node $a_{2}$ has to use the virtual arc $(o, o, 7: 00,17: 00)$ to satisfy the above standard flow balance constraints, by virtually reaching from node $o$ to node $a_{2}$ within the given travel time budget. 
Activity performingconstraints. The second set of constraints need to ensure either (i) an activity at node abeing performed with a duration of $T^{a}$ or (ii) the virtual traveling arc being selected.In Eq. (3), accordingly, we introduce a coefficient $g_{i, j, t, s}(o, a, \tau)$ to indicate condition (i) asingle activity-performing arc $\left(a, a, t, t+T^{a}\right)$ is selected at the activity node $a$, or (ii) the virtual traveling arc is used.

$$
\sum_{(i, j, t, s) \in A}\left[g_{i, j, t, s}(o, a, \tau) \times x_{i, j, t, s}(o, a, \tau)\right]=1, \text { for all } o, a \in P, \tau \in H
$$

Eq. (3) can be expressed as $x_{o, o, \tau, \tau+T(o, a)}(o, a, \tau)+\sum_{t \in H} x_{a, a, t, t+T^{a}}(o, a, \tau)=1$, where $x_{o, o, \tau, \tau+T(o, a)}(o, a, \tau)$ corresponds to the virtual traveling arc, and $\sum_{t \in H} x_{a, a, t, t+T} a(o, a, \tau)$ indicates the set of activity-performing arcs.

Coupling constraints between space-time arcs and physical links. To characterize theconnection between the physical link and space-time network arc representation, we introduce inequality (4)to express the relationship between the selection of a physical link and potential time-dependent arcs.

$$
x_{i, j, t, s}(o, a, \tau) \leq y_{i, j} \text {, for all } o, a \in P, \tau \in H,(i, j, t, s) \in A,(i, j) \in E^{B}
$$

Construction budgetconstraint. Construction cost is another important factor in the transportation network design problem. Typically, the total construction cost cannot exceed the predetermined financial budget $K$.

$$
\sum_{(i, j) \in E^{B}}\left[f_{i, j} \times y_{i, j}\right] \leq K
$$

There are also binary definitional constraints for variables $x$ and $y$.

$x_{i, j, t, s}(o, a, \tau) \in\{0,1\}$, for all $o, a \in P, \tau \in H,(i, j, t, s) \in A$

$y_{i, j} \in\{0,1\}$, forall $(i, j) \in E^{B}$

Objective function. The goal of the proposed model is to design a transportation network that maximizes the accessibility of travelers, equivalently, minimizing the inaccessibility in terms of unreachable activity locations within given TTB and activity-performing constraints. The objective function to be minimized is now formally stated as Eq. (6).

$$
Z=\sum_{o, a \in P} \sum_{\tau \in H} \sum_{(i, j, t, s) \in A}\left[c_{i, j, t, s}(o, a, \tau) \times x_{i, j, t, s}(o, a, \tau)\right]
$$

That is, if a traveler can perform the activity at node $j$ departing from node $i$ at time $\tau$ within given time budget, he/she will use the existing space-time arcs whose costs $c_{i, j, t, s}(o, a, \tau)$ of zero. Whereas, if a location is beyond the reach, the individual has to virtually reach it according to the flow balance constraint (2) using avirtualarc, leading to the tour cost of1. In summary, the total number of inaccessible destinations is equivalent to the total volume on all virtual space-time traveling arcs $\sum_{o, a \in P} \sum_{\tau \in H} x_{o, o, \tau, \tau+T(o, a)}(o, a, \tau)$ (across all activity location pairs).

\section{Lagrangian relaxation-based solution algorithms}

The proposed optimization problem can be summarized as the following conceptual form, where the major decision variables $\boldsymbol{X}$ correspond to a high-dimensional vector, which could lead to computational difficulties for large-scale real-world networks.

\section{$\underline{\text { Problem P1 }}$}

$\min Z$ 
s.t. Constraints (2-5) and binary constraints for variable vectors $\boldsymbol{X}=\left[x_{i, j, t, s}(o, a, \tau)\right]$ and $\boldsymbol{Y}=\left[\mathrm{y}_{i, j}\right]$.

It is important to recognize that Problem P1 can be viewed as a combination of two problems with standard and practically efficient solution algorithms. The first one is a time-dependent shortest path problem with flow balance constraints (2), activity-performing constraint (3); the second one is a knapsack problem subject to the total construction budget constraint (5), while coupling constraints (4) link two sets of variables $\boldsymbol{X}$ and $\boldsymbol{Y}$.

Discrete network design problems with multi-commodity flows can be cast in various forms of integer or mixed integer programming models. Many related survey papers (e.g. Magnanti and Wong, 1984), have discussed the NP-hard complexity and various solution methods, such as branch and bound and valid inequalities, which have been used to address the computational challenge for solving real-world medium-scale problems. In this study, by recognizing the space-time prism can be quickly computed through a time-dependent shortest path algorithm, we adopt a Lagrangian relaxation approach to reformulate and further decompose the prime problem to a combination of two easy-to-solve problems: a constraint time-dependent shortest path problem and a knapsack problem by relaxing the coupling constraint Eq. (3). The above subproblems have very efficient solution algorithms such as label correcting and dynamic programming algorithms, which are suitable for large-scale networks typically needed for computing accessibility measures. To meet the computational challenges for real-world data sets, we reformulate and dualizethe complicating constraints (3) and (4) using Lagrangian relaxation, as shown in $\mathrm{P} 2$. By introducing activity participation multiplier $\lambda(o, a, \tau)$, and link selection multiplier $\pi_{i, j, t, s}(o, a, \tau) \geq 0$, we can construct the dualized Lagrangian function in Eq. (7).

Problem P2:

$$
\begin{gathered}
\min \mathrm{L}=\sum_{o, a \in P} \sum_{\tau \in H} \sum_{(i, j, t, s) \in A}\left[c_{i, j, t, s}(o, a, \tau) \times x_{i, j, t, s}(o, a, \tau)\right] \\
+\sum_{o, a \in P} \sum_{\tau \in H}\left\{\lambda(o, a, \tau) \times\left[1-\sum_{(i, j, t, s) \in A} g_{i, j, t, s}(o, a, \tau) \times x_{i, j, t, s}(o, a, \tau)\right]\right\} \\
-\sum_{o, a \in P} \sum_{\tau \in H} \sum_{(i, j, t, s) \in A:(i, j) \in E^{B}}\left\{\pi_{i, j, t, s}(o, a, \tau) \times\left[y_{i, j}-x_{i, j, t, s}(o, a, \tau)\right]\right\}
\end{gathered}
$$

s.t. Constraints (2) and (5) and binary constraints for $\boldsymbol{X}$ and $\boldsymbol{Y}$.

For multipliers $\hat{\boldsymbol{\lambda}}, \hat{\boldsymbol{\pi}}$ with certain values, one can further decompose P2 into two sets of subproblems Px and Py. In our approach, these values are determined through a subgradient algorithm. Besides the subgradient method, one can use other computationally efficient but apparently relatively sophisticated method such as bundle-based relaxation methods proposed by Crainic et al. (2001). Note that, there are a total of $|P| \times(|P|-1) \times|H|$ subproblemsPx, each corresponding to an individual origin-activity location pair $(o, a)$ departing at time $\tau$.

\section{$\underline{\text { Time-dependent least cost path subproblemsfor each origin-activity location pair }}$}

$\mathrm{P}_{\mathrm{X}}$ :

$\min \mathrm{L}_{\mathrm{X}}=\min \sum_{o, a \in P} \sum_{\tau \in H} \mathrm{~L}_{X(o, a, \tau)}$ 
$\min \sum_{o, a \in P} \sum_{\tau \in H}\left\{\left[\sum_{(i, j, t, s) \in A}\left(c_{i, j, t, s}(o, a, \tau)-\lambda(o, a, \tau) \times\right.\right.\right.$

$\left.\left.\left.g_{i, j, t, s}(o, a, \tau)\right)+\sum_{(i, j, t, s) \in A:(i, j) \in E^{B}} \pi_{i, j, t, S}(o, a, \tau)\right] \times x_{i, j, t, s}(o, a, \tau)+\lambda(o, a, \tau)\right\}$

s.t. constraints (2) and binary constraints for $\boldsymbol{X}$

Knapsack subproblem

Py:

$\operatorname{minL}_{\mathrm{y}}=-\sum_{o, a \in P} \sum_{\tau \in H} \sum_{(i, j, t, s) \in A:(i, j) \in E^{B}}\left[\pi_{i, j, t, s}(o, a, \tau) \times y_{i, j}\right]$

s.t. constraints (5) and binary constraints for $\boldsymbol{Y}$

According to dual optimization theory, the Lagrange multipliers, $\lambda(o, a, \tau)$ and $\pi_{i, j, t, s}(o, a, \tau)$ can also be interpreted as shadow price associated with activity time constraints, and arc-to-link mapping constraints, respectively. These Lagrange multipliers reflect the shadow prices, which are the change in the optimal value of the objective function due to the change of parameters in different constraints (Zhao et al., 1999; Yuan et al., 2005).

If we further simplify the above model without enforcing the activityconstraints, the problem Px then becomes a more straight-forward path finding problem between origin $o$ and activity location $a$, with a single set of multipliers $\pi_{i, j, t, s}(o, a, \tau)$, representing the price for travelers from $o$ to $a$ to use the possible added link $(i, j)$ in set $E^{B}$.In this case, a dynamic programming based algorithm needs to be used to solve a standard Knapsack problem (Dreyfus and Law, 1977). If the total path price, $\hat{c}(o, a, \tau)=\sum_{(i, j, t, s) \in A:(i, j) \in E^{B}}\left[\pi_{i, j, t, s}(o, a, \tau) \times x_{i, j, t, s}(o, a, \tau)\right]$, paid by the traveler from $o$ to $a$ on the physical network is less than the inaccessibility $\operatorname{cost} c_{i, j, t, s}(o, a, \tau)$ for the virtual arc, then he/she can select the newlyconstructed roads to perform the traveling activity. Otherwise, if the total path price is too high for using the road to be constructed, that is, $\hat{c}(o, a, \tau)>c_{o, o, \tau, \tau+T(o, a)}(o, a, \tau)$, then resorting to the virtual traveling arc (with the preset 1 unit of inaccessibility cost) is the only option from the cost minimization standard point.

Algorithm 2: Lagrangian relaxation-based algorithm for solving simplified problem P2 without enforcing the activity constraints

\section{Step 1: Initialization}

Set iteration number $k=0$;

Choose positive valuesto initialize the set of Lagrangian multipliers $\pi_{i, j, t, s}(o, a, \tau)$;

\section{Step 2: Solve decomposed dual problems}

Solve subproblemP $\mathrm{P}_{\mathrm{X}}$ using a standard time-dependent shortest path algorithm and find a path solution $\boldsymbol{X}(o, a, \tau)$ for each origin-activitylocation pair $(o, a)$ departing at time $\tau$;

Solve subproblemPy, for example, using a dynamic programming algorithm, to find a value for $\boldsymbol{Y}$;

Calculate primal, dual and gap valuesof P2.

Step 3: Update Lagrangian multipliers

Update Lagrangian multipliers $\quad \pi_{i, j, t, s}(o, a, \tau) \quad$ using subgradient $\pi_{i, j, t, s}(o, a, \tau)+d^{k} \times\left[x_{i, j, t, s}(o, a, \tau)-y_{i, j}\right]$, where $d^{k}$ is the step length at iteration $k$.

Step 4: Termination condition test 
If $k$ is less than a predetermined maximum iteration value, or the gap is smaller than a predefined toleration gap, terminate the algorithm; otherwise $k=k+1$ and go back to Step 2 .

We refer interested readers to the papers by Ziliaskopoulos and Mahmassani (1993) and Pallottino and Scutellà (1998) for more information on space-time network construction and computationally time-dependent shortest path algorithms. Yang and Zhou (2014) also has a recent study on using a Lagrangian relaxation algorithmic framework for urban road networks, with a more detailed discussion on various subgradient updating rules.

\section{Applying the network accessibilitydesign model in practice}

After presenting the mathematical models, we also need to examine a number of practically importantissues in applying the proposed network design model in planning practices.

\subsection{Comparing accessibility with other commonly used network design measures}

It should be remarked that, the accessibility measure is closely related to the commonly used connectivity measure, which typically highlights if a pair of one origin and one destination is connected given static or time variant travel times in a network. If an activity location is accessible, then it must be connected from the origin physically. Compared to the standard connectivity measure, our proposed accessibility measure needs to consider additional three inputs of (1) time-varying congestion levels associated with specific departure times at the origin and activity locations; (2) total travel time budget and (3) required activity duration. The final number of accessible activity locations is sensitive to any of the above inputs. Due to the travel time budget constraint, not all connected activity locations are reachable. Under congestion circumstances, the potential accessible area will shrink in size on account of low travel speed even its connecting locations are the same.

As we can see from the literature, the existing use of the connectivity measure in network design models is mainly for traffic evacuation applications that needs to transport evacuees from their current positions to shelters, while the accessibility plays a more active role in urban planning, especially for widely used Activity Based Models (ABM) concerning about the household activity scheduling, potential opportunities to reach valuable destinations and within the total travel time budgets (Miller, 2014).

Level of Service(LOS), as one of common network design criteria, is typically quantified by either link density or speed, which can be further mapped to a specific value of travel time at different times of a day (Ghiani et al., 2003). As we discussed before, the dynamic travel time performance can be incorporated to the accessibility measures for different specific departure times in Eq. (1).

\subsection{Preparing time-dependent travel time data}

There are multiple methods to prepare time-dependent travel time data. One can use observed travel time at different times of a day as the input to our model, e.g. using data from mobile phones or other traffic probe sources. One potential challenging modeling issue is that, for those candidate links to be built, the exact time-dependent travel times are difficult to obtain unless a more sophisticated dynamic traffic assignment process is used with restrictive user equilibrium constraints. To overcome this challenge, one can adapt a "predicted mobility" method (El-Geneidy and Levinson, 2006), that is, use a default profile of time-dependent speed for different classes of road to generate the likely 
time-dependent travel times for candidate links, without requiring a large number of restrictive assumptions and extra computation efforts for dynamic traffic assignment.

For multi-modal network design problems, one can also use predetermined transit timetables to establish time-sensitive transit/train schedules used in the proposed space-time network. Interested readers can find a detailed study by Tasic et al. (2014) on the impact of acceptable walking time, available time budget and transit schedule variability, and the time-varying number of transit stops reachable at different departure times.

\subsection{Handling uncertain or elastic demand}

For travelers, high accessibility means that they may have the ability to reach dozens or hundreds of destinations in a given time budget, but they can only travel to one of them at each time. Under the activity-based conceptual framework, travel demand occurs to meet the specific needs to be at particular places at specific times to conduct different purposes activities including work, shopping, recreation (Mokhtarian and Salomon, 2001). Therefore, travel demand is assumed to be fixed in the proposed accessibility model although improvingnetwork accessibility could lead to induced travel demand. This suggests that our model should be used with some caution for roads and highway improvements only. However, our method could also be used for travel demand management strategies (Litman, 2003; Handy, 2005). For example, one can easily incorporate many important design factors for transit-oriented development (TOD) (Tasic et al. 2014), e.g., adding stops for reducing the walking times, and adding transit frequency, each corresponding to various spatial and temporal elements in the proposed space-time structures.

\subsection{Accessibility-based network design strategies}

For urban transportation network design problems, network accessibility can be improved mainly in three ways: construction, travel behavior/activity time duration and land use. Table 5 shows the solution categories, specific strategies and the related variables in Eq. (1). First, from construction point of view, reducing travel time and providing other travel options are two major strategies that can be adopted by planners. Different from the mobility-based concept, the essential goal of reducing travel time strategy, including transportation facility capacity expansion and new road construction, is also able to increase the ease of end-to-end accessibility. The other strategy is providing more travel options to travelers, such as public transit services, bicycle lines and pedestrian lines under certain situations. Table 5 further summarizes different solution strategies that can be incorporated in the proposed accessibility-oriented network design and traveler active demand management framework as an overarching optimization model.

\section{(Insert Table 5)}

Overall, the above measures aim to reduce the total time spent on the road, and therefore provide more activity locations that are accessible within the same travel time budget.

\subsection{Possible ways to consider congestion effects through additional capacity constraints}

One of the most challenging questions in our research is how to fully consider congestion effects associated with road capacity constraints. One can first change the binary state variable to $x_{i, j, t, s}(k)$ to represent the arc selection by agent $k$ belonging to origin-activity location pair set $D(o, a, \tau)$. Then 
the sum of all agents using the space-time arc need to satisfy the time-dependent road capacity $\operatorname{cap}(i, j, t, s)$ on arc $(i, j, t, s)$. Compared to Eq. (4), where we only consider links to be built, Eq. (10) are used to consider all links in the network, and dualizing this set of constrains will introduce a new set of link multipliers $\rho(i, j, t, s)$ for each arc $(i, j, t, s)$. These new multiplierscan be added into the Lagrangian relaxation function Eq. (7) and utilized in a subgradient type algorithm. By doing so, onecan help traveling agents select the less congested routes/links and reach a certain state of system optimal or more complex user equilibrium conditions, through a point queue model or more sophisticated spatial queue model, as shown by one recent study by Ma et al. (2015).

$$
\sum_{o, a \in P} \sum_{\tau \in H} \sum_{k \in D(o, a, \tau)} x_{i, j, t, s}(k) \leq\left[\operatorname{cap}(i, j, t, s) \times y_{i, j}\right], \text { for }(i, j, t, s) \in A,(i, j) \in E
$$

On the other hand, we can see adding a new set of multipliers for all links can better capture various real-world constraints but could further complicate the proposed solution framework. We will examine the related algorithmic and computational performance issues in our future study.

\section{Numerical experiments}

This section evaluates the sensitivity of the accessibility-optimal solutions under different conditions, and demonstrate the effectiveness of the proposed method in modeling real-world networks.

\subsection{Illustrative examples}

Using the hypothetic three-node network in Fig. 7 with the cost data in Table 6, we are interested in how the proposed model is sensitive todifferent decision factors and criteriain terms ofoverall accessibility.

\section{(Insert Table 6)}

The network design problem is implemented and solved bya commercial solution solver, General Algebraic Modeling System (GAMS) (Rosenthal, 2004).

Impact of construction budget. As shown in Table 7, if there are more available budgetary resources, the number of links that can be built increases, which leads to more accessible origin-activity location pairs.Essentially, providing more travel options through transportation facility capacity expansion and new roads construction can increase the end-to-end accessibility. It is interesting to see that, under scenario no. 3, an one-way loop of links are selected along a single direction, namely $\left(o, a_{1}\right),\left(a_{1}, a_{2}\right),\left(a_{2}, o\right)$ or $\left(o, a_{2}\right),\left(a_{2}, a_{1}\right),\left(a_{1}, o\right)$. Either solution (with the same construction cost)can enable the access for all 6 origin-activity location pairs. When the construction budget is further increased, say from 9 units to 10units or 18 units, the system-wide accessibility measure keeps unchanged, with shorter travel times between origin-activity location pairs, which is not modeled in the proposed accessibility-maximization program.

(Inset Table 7)

From the above example, it is important to recognize the significance of accessibility-maximal solutions: this generates an exact upper bound or benchmark for attainable accessibility gains, with possible network design scenarios. Such optimality information is very useful for further evaluating different solutions generated from the planning-oriented approach. It should be also remarked that, 
additional construction budget could also providemore non-driving travel options, such as public transit service, bicycle lines and pedestrian lines, which also meet the system accessibility goal.

Impact of travel time budget. In this set of experiments, we set the total available construction cost to 10 units, which would allow building at most 4 links. In Table 8, we vary the total travel time budget with a fixed activity duration of 2 units. It is interesting to see that, when the travel time budget is very tight, the optimal solutions, e.g. under scenario 2, do not suggest to build all road links and still have unused construction budget resources. The result is expected, as building additional links under this case does not lead to an increase in the system-wide accessibility givenvery restrictedTTB constraint. Practically, this implies that, the accessibility-enhancement strategies have to be carefully planned and coordinated, under both TTB and construction cost constraints. Otherwise, some certain scenarios could lead to cost-ineffective solutions.

(Inset Table 8)

In general, it is difficult to increase the acceptable total travel time budget, especially under heavy congestion. Alternatively, planners can consider options to reduce the activity duration or shift departure times, e.g. using flexible working hours, to enhance transportation network accessibility, without requiring additional construction monetary budgets.

Impact of link travel time changes. Table 9 shows different scenarios with different sets of link travel time values. The general implication is that, the change in the link travel times, e.g., due to traffic congestion, or congestion mitigation strategies, or fast transit service, could dramatically affect the results of network design solutions. As the proposed formulation is built on a time-dependent network, one can easily consider dynamic travel times, so a number of emerging active traffic demand management strategies, such as demand spreading,time of day tolling (affecting generalized link travel times), can be adopted to enhance the accessibility at different time periods.In addition, many land use policies that encourage reasonable activity location density and functional facility distribution, could also increase the proximity of common destinations. From the perspective of the proposed network design model, such policies could reduce the amount or distance of travel while maintaining a desirable level of personal transportation accessibility.

(Inset Table 9)

\subsection{Illustration of Lagrangian multiplier}

Using the six-node network shown in Fig. 8, we illustrate how to solve the transportation network design problem P1 with the proposed Lagrangian relaxation algorithm. In this network, solid lines denote the existing links, and the dashed lines are all the candidate links to be built with link labels as the corresponding travel time. Given $\mathrm{TTB}=3$ time units, we consider only one origin-activity location pair $(1,4)$ with three links to be selected for the final construction, $\left|E^{B}\right|=3$. Obviously, the 3-link optimal solution covers links $(1,5),(5,6)$ and $(6,4)$, with a feasible and optimalvalue for the objective function as zero (the single origin-activity pair is accessible). This also gives a feasible upper bound (UB) of the optimal solution as zero. For any vector of the Lagrangian multipliers, the value of the 
Lagrangian function is a lower bound(LB) on the optimal objective function value of the original optimization problem, which corresponds to Eq. (7) in the proposed model.

Table 10 lists the value of Lagrangian multipliers at each iteration. At the first iteration, we start with zeromultipliers $\pi_{i, j}$. The shortest path from node 1 to node 4 is the sequence of $1->5->6->4$. The subsequentsubgradient algorithm then accordingly sets the prices of all three passing links to 1 . Under this situation, the least cost path finding algorithm has to use the virtual link from 1 to 4 , as the total Lagrangian pricesfor the feasible path is 3 , which is higherthan the value of 1 on the virtual link. This leads to $\mathrm{Lx}=1$. On the other hand, (for the same values of multipliers) the knapsack subproblem problem wants to build three links on path $1->5->6->4$, leads to the Lagrangian pricesthat is expected to collect as $3, \mathrm{Ly}=-3$, the $\mathrm{LB}=1-3=-2$. As $\mathrm{UB}=0$, the optimality gap $=\mathrm{UB}-\mathrm{LB}=2$. As no one uses the links to be built, the subgradient algorithm further helps to lower the total Lagrangian pricesof those three links to $0.4 \times 3=1.2$ and $0.3 \times 3=0.9$ at iterations 3 and 4 . In this case, flow from 1 to 4 returns back to the physical network, as the cost of the physical path $=0.9<1$ (virtual traveling). One can easily verify that $\mathrm{Lx}=0.9$ and $\mathrm{Ly}=0.9$. Because $\mathrm{LB}=\mathrm{UB}=0$, the optimality gap $=0$ and the optimal solution is found.

(Insert Fig. 8)

(Inset Table 10)

\subsection{Medium-scale experiments}

A simplified Sioux Falls network consisting of 24 nodes and 76 directional links is shown in Fig.9. The free-flow link travel times are used in the experiment, and there are a total of $24 \times 23=552$ origin-activity location pairs. In this hypothetic example, we consider to select $K$ out of 10 directional optional links for future construction.

(Insert Fig. 9)

(Insert Fig. 10)

(Insert Fig. 11)

Fig. 10 shows the iteration-by-iteration converging patterns of upper bounds and lower bounds generated from the proposed algorithm, when changing TTB from 16 to $19 \mathrm{~min}$, with $K=3$. Typically, the lower bound estimates are improved significantly during the first few iterations, and the duality gap between the upper bound (obtained from the objective function of primal feasible solutions) and the lower bound estimates can be reduced dramatically to a relatively small difference after a certain number of iterations. Fig. 11 further quantifies the relative optimality gap=(UB-LB)/UB under different cases of link selection size $K$. We obtain less than $4 \%$ gaps in all three cases, but the non-zero gaps still existdespite 40 iterations. This behavior can be explained by the inherent limitation of Lagrangian lower bound estimation techniques and subgradient algorithms. Interested readers are referred to Yang and Zhou (2014) and Zhao et al. (1999) for more discussionson the solution quality for space-time networks and alternative subgradient step size selection rules. 


\subsection{Large-scale experiments}

Obviously, the time-dependent least cost shortest path algorithm is still the most computational consuming step of the proposed algorithm. Our algorithm is implemented in $\mathrm{C}++$ with a parallel computing capability to utilize multi-core CPUs. We now test the computational performance of the proposed algorithm on the large-scale Chicago sketch network, shown in Fig. 12. This network is an aggregated representation of the Chicago region with 387 zones and 1.2 million vehicles during a peak hour.

(Insert Fig. 12)

To prepare this data set, we obtain the corridor-specific time-dependent travel times from the website maintained by RoadStatsInc. (http:/www.travelmidweststats.com/), which reports the historical travel time for the Chicago metropolitan area, Illinois. For freeway corridors without the travel time data, we use a default regional average travel time profile based on the sample corridor shown in Fig. 13, where the travel time indices (average travel time divided by free-flow travel time) of three sample corridors in Chicago area are listed based on the observed average travel time. The arterial street travel times are obtained from a dynamic traffic simulation (Zhou and Taylor, 2014) using time-dependent OD demand. We need to calculate time-dependent shortest path for 149,382 origin-activity location pairs at each iteration. This experiment uses 70-minute TTB departing between time period from6:00 AMto 11:00 AM, and we plan to select 5 out of 20 candidate links. The algorithm is evaluated on a Dell Precision T7610 Workstation with $201.9 \mathrm{GHz}$ CPU cores and 192GB

RAM. There are 40 computational threads available on this computer, so the multiple origin-activity location-specific shortest path calculation tasks can be easily decomposed into different CPU threads, thanks to the proposed scalable Lagrangian relaxation framework.

(Insert Fig. 13)

(Insert Table 11)

Shown in Table 11, it takes 68 minutes of CPU time, approximately 1.7 minutes per iteration, to find a reasonably good network design solution within a $0.1 \%$ gap for 40 iterations. For a workstation with only a single CPU thread, then it might take around 45 CPU hours for completing similar calculation, which highlights the benefits and needs for enabling the parallel computing paradigm for the proposed algorithm. From Table 11, it is also interesting to observe that, there is no significant improvement in solution quality after 10 iterations and we reach a $4 \%$ gap after the first 5 iterations.Fig. 14 illustrates the network-wide accessibility level from the experimental result of the optimal network design solution and the related increased accessibility measure at each activity location. This heat map indicates that, a location is considered to have high accessibility level iftravelers from this location are able to reach more activity locations within the given travel time budget.One can easily observe that, the downtown Chicago area still has the highest accessibility as a natural hub connecting to different freeway facilities. With such a visualization tool, one can further examine the specific impact of enhanced accessibility at different areas associated with added links.

(Insert Fig. 14) 
In general, the case we consider has not fully taken into account the complex congestion effect caused by road capacity constraints, so the selecting a few links from 20 candidate links is in fact a relatively simple process. The close-to-optimal solutions can be found in a few iterations, as the numbers of origin-activity location pairs using the candidate links become stabilized quickly and no further significant solution quality improvement can be made. It should be also remarked that, if a more complex time-dependent capacity constraint is considered or the realistic user equilibrium behavior is incorporated, then how to select candidate links to build is not a trivial task as the route choices under congestion are difficult to mathematically model in its own right (e.g. through a bi-level programming framework).

\section{Concluding remarks}

Accessibility is an important performance measure which is often neglected in transportation network design models. This paper presents a linear integer programming model to maximize end-to-end accessibility within space-time constraints. A time-dependent network representation and a Lagrangian relaxation based solution method are then developed to solve this problem in real-world applications. The developed iterative search algorithm can provide a systematic algorithmic framework to quantify the quality of solutions. Using a number of illustrative and real-world examples, our proposed urban transportation network design problem for maximizing network accessibility can be effectively used to evaluate a wide range of practically important policies and scenarios, such as road construction, changing travel behavior/activity time duration, congestion mitigation as well as long-term land use options.

Compared to many standard space-time network construction methods, such as detailed procedures described by Ziliaskopoulos and Mahmassani (1993) and Pallottino and Scutellà (1998), our proposed method has added a few new features to capture characteristics of accessibility-oriented network design problems. 1) Virtual traveling arcs are introduced to better incorporate travel time budget constraints within a space-time network, and further represent the inaccessible results between an origin-activity pair. 2) Activity performing arcs are added at activity locations to reflect the required activity participation time.

Compared to a recent study by Yang and Zhou (2014), our paper also uses space-time networks as the foundation for mathematical modeling and adopts a general Lagrangian relaxation solution methodology. Yang and Zhou (2014) mainly consider to how to relax non-anticipatory constraints in a stochastic routing setting, and the subproblems involve the shortest path problem and an auxiliary-variable optimization problem specifically designed for linking different scenarios. In our research, we are mainly concerned about a class of joint flow routing and resource allocation problems, the coupling constraint can be dualized to decouple the routing and resource allocation subproblems, each corresponding to an efficient solution algorithms.

Our contributions in this paper focus on incorporating space-time accessibility into network design models. As an initial step, we necessarily neglect several real-world aspects of transportation networks; these include elasticity of travel demand and modal split, stochastic events and long-term network flow equilibrium (Chen et al, 2011; Crainic et al., 2011;Anand Hong, 2014; Liu et al., 2015). Therefore, the techniques in this paper are best suited for short planning horizons (1-3 years) since a change in accessibility may affect travel demands, modal split and network flow patterns. Section 6 discusses how to resolve some of these issues in practice. Future research will be directed to capturing 
these real-world complexities directly into accessibility-oriented network design models. For example, one possibility is a two-stage stochastic programming framework in which at the first stage a subset of transportation links or services is selected to be built and recourse traveler routing decisions need to made in the second stage according to realized demand scenarios.

In our future research, we will integrate our proposed model into traditional transportation planning methods and large-scale real-world regional networks, focusing on improving multi-facet system performance, in addition to the widely used mobility-oriented planning perspective. We also recognize that, although planning for mobility will generally increase the network accessibility, it would be interesting to study how accessibility-based planning methods could further expand the range

of potential intelligent transportation management solutions, without requiring road construction resources.

\section{Acknowledgements}

The material in this paper is based on research supported by National Science Foundation under grant No. BCS-1224102 "Measuring the Environmental Costs of Space-time Prisms in Sustainable Transportation Planning". The research of the first author is carried out when she is a visiting student at Arizona State University and supported by Chinese Scholarship Council. 


\section{References}

Aguado, J. S., 2009. Fixed charge transportation problems: a new heuristic approach based on Lagrangean relaxation and the solving of core problems. Annals of Operations Research172 (1), 45-69.

An, K., Hong, K. L., 2014. Ferry service network design with stochastic demand under user equilibrium flows. Transportation Research Part B66 (8), 70-89.

Ben-Akiva, M. E., Lerman, S. R., 1979. Disaggregate travel and mobility choice models and measures of accessibility. Behavioural travel modelling, 654-679.

Ben-Akiva, M. E., Lerman, S. R., 1985. Discrete choice analysis: theory and application to travel demand. MIT Press, Cambridge, Mass.

Bowman, J. L., Ben-Akiva, M. E., 2001. Activity-based disaggregate travel demand model system with activity schedules. Transportation Research Part A35 (1), 1-28.

Chen, A., Yang, C., Kongsomsaksakul, S., Lee, M., 2007. Network-based accessibility measures for vulnerability analysis of degradable transportation networks. Networks and Spatial Economics 7 (3), 241-256.

Chen, A., Zhou, Z., Chootinan, P., Ryu, S., Yang, Wong, S. C., 2011. Transport network design problem under uncertainty: a review and new developments. Transport Reviews31(6), 743-768.

Chen, S., Claramunt, C., Ray, C., 2014. A spatio-temporal modelling approach for the study of the connectivity and accessibility of the Guangzhou metropolitan network. Journal of Transport Geography36, 12-23.

Chen, Y., Ravulaparthy, S., Deutsch, K., Dalal, P., Yoon, S. Y., Lei, T., Pendyala, R. M., Bhat, C. R., Hu, H. H., 2011. Development of indicators of opportunity-based accessibility. Transportation Research Record: Journal of the Transportation Research Board2255, 58-68.

Crainic, T. G., 2000. Service network design in freight transportation. European Journal of Operational Research122 (2), 272-288.

Crainic, T. G., Frangioni, A., Gendron, B., 2001. Bundle-based relaxation methods for multicommodity capacitated fixed charge network design. Discrete Applied Mathematics112 (1), 73-99.

Crainic, T., Fu, X., Gendreau, M., Rei, W., Wallace, S., 2011. Progressive hedging-based metaheuristics for stochastic network design. Networks 58 (2), 114-124.

Curtis, C., Scheurer, J., 2010. Planning for sustainable accessibility: Developing tools to aid discussion and decision-making. Progress in Planning74(2), 53-106.

Daganzo, C., 2010. Structure of competitive transit networks. Transportation Research Part B44 (4), $434-446$.

Dantzig, G., 1963. Linear programming and extensions. Princeton University Press, Princeton, N.J.

Dong, X., Ben-Akiva, M. E., Bowman, J. L., Walker, J. L., 2006. Moving from trip-based to activity-based measures of accessibility. Transportation Research Part A40 (2), 163-180.

Dreyfus, S. E., Law, A. M., 1977. The art and theory of dynamic programming. Academic Press, New York.

El-Geneidy, A.M., Levinson, D.M., 2006. Access to destinations: development of accessibility measures. Minnesota Department of Transportation.

Erlander, S., 1977. Accessibility, entropy and the distribution and assignment of traffic. Transportation Research11 (3), 149-153.

Farahani, R. Z., Miandoabchi, E., Szeto, W. Y., Rashidi, H., 2013. A review of urban transportation network design problems. European Journal of Operational Research229 (2), 281-302. 
Geertman, S. C., Ritsema Van Eck, J., 1995. GIS and models of accessibility potential: an application in planning. International Journal of Geographical Information Systems9 (1), 67-80.

Ghiani, G., Guerriero, F., Laporte, G., Musmanno, R., 2003. Real-time vehicle routing: Solution concepts, algorithms and parallel computing strategies. European Journal of Operational Research151 (1), 1-11.

Guihaire, V., Hao, J., 2008. Transit network design and scheduling: a global review. Transportation Research Part A42 (10), 1251-1273.

Handy, S., 2005. Planning for accessibility: In theory and in practice. Access to destinations, 131-147.

Hansen, W. G., 1959. How accessibility shapes land use. Journal of the American Institute of Planners25 (2), 73-76.

Hou, Q., Li, S., 2011. Transport infrastructure development and changing spatial accessibility in the Greater Pearl River Delta, China, 1990-2020. Journal of Transport Geography19 (6), 1350-1360.

Hägerstrand, T., 1970. What about people in Regional Science?.Papers of the Regional Science Association 24 (1), 6-21.

Jiang, Y., Szeto, W. Y., 2015. Time-dependent transportation network design that considers health cost. Transportmetrica A: Transport Science 11 (1), 74-101.

Kang, J.E., Chow, J.Y.J., Recker, W.W., 2013. On activity-based network design problems. Transportation Research Part B57, 398-418.

Kennington, J., Nicholson, C., 2010. The uncapacitated time-space fixed-charge network flow problem: an empirical investigation of procedures for arc capacity assignment. INFORMS Journal on Computing22 (2), 326-337.

Litman, T., 2003. Measuring transportation: traffic, mobility and accessibility. ITE Journal, 73 (10), 28-32.

Litman, T., 2011. Evaluating accessibility for transportation planning. Victoria, BC: Victoria Transport Policy Institute.

Liu, H., Wang, D., 2015. Global optimization method for network design problem with stochastic user equilibrium. Transportation Research Part B72, 20-39.

Luathep, P., Sumalee, A., Lam, W., Li, Z., Lo, H., 2011. Global optimization method for mixed transportation network design problem: A mixed-integer linear programming approach. Transportation Research Part B45 (5), 808-827.

Ma, J., Smith, B., Zhou, X., 2015. Personalized real-time traffic information provision: Agent-based optimization model and solution framework. Transportation Research Part C. In Press.

Magnanti, T. L., Wong, R. T., 1984. Network design and transportation planning: models and algorithms. Transportation Science 18 (1), 1-55.

Miandoabchi, E., Daneshzand, F., Szeto, W. Y., Farahani, R. Z., 2013. Multi-objective discrete urban road network design. Computers and Operations Research40 (10), 2429-2449.

Miller, H. J., 1991. Modelling accessibility using space-time prism concepts within geographical information systems. International Journal of Geographical Information Systems5 (3), 287-301.

Miller, H. J., 1999. Measuring space-time accessibility benefits within transportation networks: basic theory and computational procedures. Geographical Analysis31 (1), 1-26.

Miller, H. J., 2005. A measurement theory for time geography. Geographical Analysis37 (1), 17-45.

Miller, H. J., 2014. Activity-based analysis. In Handbook of Regional Science 705-724. Springer Berlin Heidelberg. 
Mokhtarian, P. L., Salomon, I., 2001. How derived is the demand for travel? some conceptual and measurement considerations. Transportation Research Part A35 (8), 695-719.

Murawski, L., Church, R. L., 2009. Improving accessibility to rural health services: the maximal covering network improvement problem. Socio-Economic Planning Sciences43 (2), 102-110.

Neuberger, H., 1971. User benefit in the evaluation of transport and land use plans. Journal of Transportation Economics and Policy5(1)52-75.

Ortega, F., Wolsey, L. A., 2003. A branch-and-cut algorithm for the single-commodity, uncapacitated, fixed-charge network flow problem. Networks41 (3), 143-158.

Pallottino, S., Scutellà, M. G., 1998. Shortest path algorithms in transportation models: classical and innovative aspects. Equilibrium and Advanced Transportation Modelling 245-281. Springer US.

Recker, W. W., 2001. A bridge between travel demand modeling and activity-based travel analysis. Transportation Research Part B35 (5), 481-506.

Rosenthal, R. E., 2004. GAMS--a user's guide.

Santos, B., Antunes, A., Miller, E. J., 2008. Integrating equity objectives in a road network design model. Transportation Research Record: Journal of the Transportation Research Board2089, $35-42$.

Small, K. A., Rosen, H. S., 1981. Applied welfare economics with discrete choice models.Econometrica49 (1), 105-30.

Sohn, J., 2006. Evaluating the significance of highway network links under the flood damage: an accessibility approach. Transportation Research Part A40 (6), 491-506.

Tasic, I., Zhou, X., Zlatkovic, M., 2014. Use of spatiotemporal constraints to quantify transit accessibility: case study of potential transit-oriented development in West Valley City, Utah. Transportation Research Record: Journal of the Transportation Research Board2417, 130-138.

Taylor, M. A., 2008. Critical transport infrastructure in urban areas: impacts of traffic incidents assessed using accessibility-based network vulnerability analysis. Growth and Change39 (4), 593-616.

Ukkusuri, S. V., Mathew, T. V., Waller, S. T., 2007. Robust transportation network design under demand uncertainty. Computer-Aided Civil and Infrastructure Engineering 22 (1), 6-18.

Vickerman, R. W., 1974. Accessibility, attraction, and potential: a review of some concepts and their use in determining mobility. Environment and Planning A6 (6), 675-691.

Viswanath, K., Peeta, S., 2003. Multicommodity maximal covering network design problem for planning critical routes for earthquake response. Transportation Research Record: Journal of the Transportation Research Board1857, 1-10.

Wachs, M., Kumagai, T. G., 1973. Physical accessibility as a social indicator. Socio-Economic Planning Sciences 7 (5), 437-456.

Waddell, P., 2002. Urbansim: modeling urban development for land use, transportation, and environmental planning. Journal of the American Planning Association68 (3), 297-314.

Wang, S., Meng, Q., Yang, H., 2013. Global optimization methods for the discrete network design problem. Transportation Research Part B50, 42-60.

Weibull, J. W., 1976. An axiomatic approach to the measurement of accessibility. Regional Science and Urban Economics6 (4), 357-379.

Weibull, J. W., 1980. On the numerical measurement of accessibility. Environment and Planning A 12 (1), 53-67. 
Williams, H. C., 1976. Travel demand models, duality relations and user benefit analysis. Journal of Regional Science 16 (2), 147-166.

Williams, H. C., 1977. On the formation of travel demand models and economic evaluation measures of user benefit. Environment and Planning A9 (3), 285-344.

Wilson, A. G., 1971. A family of spatial interaction models, and associated developments. Environment and Planning A3 (1), 1-32.

Wu, Y. H., Miller, H. J., 2001. Computational tools for measuring space-time accessibility within dynamic flow transportation networks. Journal of Transportation and Statistics4(2/3), 1-14.

Yang, L., Zhou, X., 2014. Constraint reformulation and a Lagrangian relaxation-based solution algorithm for a least expected time path problem. Transportation Research Part B59 (1), 22-44.

Yuan, J., Li, Z., Yu, W., Li, B., 2005. A cross-layer optimization framework for multicast in multi-hop wireless networks. In Wireless Internet, 2005. Proceedings. First International Conference on 47-54. IEEE.

Zhao, X., Luh, P. B., Wang, J., 1999. Surrogate gradient algorithm for Lagrangian relaxation. Journal of Optimization Theory and Applications 100 (3), 699-712.

Ziliaskopoulos, A. K., Mahmassani, H. S., 1993. Time-dependent, shortest-path algorithm for real-time intelligent vehicle highway system applications. Transportation Research Record: Journal of the Transportation Research Board1408, 94-104.

Zhou, X., Taylor, J., 2014. DTALite: A queue-based mesoscopic traffic simulator for fast model evaluation and calibration. Cogent Engineering1 (1), 961345. 


\section{List of Tables}

Table 1 Comparison of key elements in transportation network design models

Table 2 Subscripts and parameters used in mathematical formulations

Table 3 Decision variables used in mathematical formulations

Table 4 Travel time data in hypothetic 3-node network

Table 5 Strategies to increase accessibility

Table 6 Travel time and construction cost data

Table 7 Impact of construction budget

Table 8 Impact of travel time budget (under 10-unit construction budget)

Table 9 Impact of link travel time (under 10-unit construction budget and 10-unit travel time budget)

Table 10 Evolution of Lagrangian multipliers for all candidate links

Table 11 Computational statistics for Chicago sketch network 


\section{List of Figures}

Fig 1. Illustration of physical network and space-time network (used in transportation network modeling literature)

Fig 2. Illustration of a space-time path (adapted from Hägerstrand 1970)

Fig 3. An illustration of accessibility concept from origin node to other nodes

Fig 4. Accessible vs. inaccessible nodes for road users with traveling time constraints

Fig 5. A simple space-time prism (Miller, 2005)

Fig 6. Mapping of accessible/ inaccessible locations with respect to potential activity area

Fig 7. An illustration of adding a link in physical network

Fig 8. An illustration of adding links in physical network

Fig 9. Simplified Sioux Falls network with 10 directional candidate links

Fig 10. Evolution of UB and LB values for different travel time budgets (each time series is labeled as LB_TTB or UB_TTB for TTB $=19,18$, and 16 min)

Fig 11. Evolution of relative optimality gap ((UB-LB)/UB) travel time budget $\mathrm{TTB}=12$ min, number of links to be selected $\mathrm{K}=4,5$ and 6

Fig 12.Chicago sketch network with 933 nodes, 2950 links, 387 zones and 20 (hypothetic) candidate links to be built

Fig 13. Sample corridor-level travel times across a day (travel time index $=$ travel time/free-flow travel time)

Fig 14. Accessibility heat map from the Chicago numerical testing results at 8:00 AM (the size of nodes reflects the additional number of activity locations accessible) 
Table 1 Comparison of key elements in transportation network design models

\begin{tabular}{|c|c|c|c|c|}
\hline Problem type & Demand & Objective functions & $\begin{array}{l}\text { Solution } \\
\text { algorithm }\end{array}$ & Publication \\
\hline \multirow{3}{*}{ MCND } & \multirow{3}{*}{ Given } & \multirow{3}{*}{ Minimizing both design costs and flow costs } & BB & \multirow{3}{*}{ Magnanti and Wong (1984) } \\
\hline & & & $\mathrm{BD}$ & \\
\hline & & & LL & \\
\hline MCND & Given & Minimizing both design costs and flow costs & -- & Crainic (2000) \\
\hline PMCND & Given & Minimizing both design costs and flow costs & -- & Crainic(2000) \\
\hline UFC & Given & Minimizing both design costs and flow costs & $\mathrm{BC}$ & Ortega and Wolsey (2003) \\
\hline RNDP & Uncertain & $\begin{array}{l}\text { Minimizing the expected and the standard } \\
\text { deviation of total system travel time }\end{array}$ & GA & Ukkusuri et al. (2007) \\
\hline SCND & Given & Minimizing both design costs and flow costs & LR, BC & Aguado (2009) \\
\hline TSFC & Given & Minimizing both design costs and flow costs & LPR & $\begin{array}{l}\text { Kennington and Nicholson } \\
(2010)\end{array}$ \\
\hline MNDP & Given & Minimizing total travel time & CCA & Luathep et al. (2011) \\
\hline ABNDP & Given & $\begin{array}{l}\text { Minimizing both design costs and } \\
\text { activity-related disutility }\end{array}$ & DH & Kang et al. (2013) \\
\hline DRNDP & Given & $\begin{array}{l}\text { Maximizing increase in consumer surplus and } \\
\text { reduction in health cost }\end{array}$ & $\begin{array}{l}\mathrm{ABC} \\
\mathrm{FWA}\end{array}$ & Jiang and Szeto (2014) \\
\hline TMNDP & $\begin{array}{l}\text { Not required, } \\
\text { focusing on } \\
\text { major activity } \\
\text { location pairs }\end{array}$ & Maximize space-time accessibility & LR & $\begin{array}{l}\text { This paper by Tong et al. } \\
\text { (2015) }\end{array}$ \\
\hline
\end{tabular}

Problem type: ABNDP—activity-based network design problem; DRNDP—discrete road network design problem; MCND—capacitated multi-commodity network design problem; MNDP—-mixed (continuous/discrete) network design problem; PMCND - path-based multi-commodity capacitated network design; RNDP—robust network design problem; SCND — capacitated single-commodity network design problem; TMNDP - time-constraint multi-commodity network design; TSFC - time-space fixed charge network design problem; UFC — uncapacitated fixed-charge network flow problem.

Solution algorithm: $\mathrm{ABC}$ - artificial bee colony algorithm;BB — branch and bound;BC — branch-and-cut; $\mathrm{BD}$ - benders decomposition; $\mathrm{CCA}$ — cutting constraint algorithm; $\mathrm{DH}$-decomposition heuristic algorithm; FWA—Frank-Wolfe algorithm; GA—genetic algorithm; LL—Lagrangian and linear programming; LPR—linear programming relaxation; LR—Lagrangian relaxation; PD—primal-dual method. 
Table 2 Subscripts and parameters used in mathematical formulations

\begin{tabular}{|c|c|}
\hline Symbol & Definition \\
\hline$N$ & Set of nodes in physical network \\
\hline$P$ & Set of activity location nodes in physical network, $P \in N$ \\
\hline E & Set of transportation links in physical network \\
\hline$E^{B}$ & Set of transportation links to be built in physical network \\
\hline$E^{E}$ & Set of transportation links existing in physical network \\
\hline$H$ & Set of time stamps in the planning horizon \\
\hline$Q$ & Set of vertexes in space-time network \\
\hline$A$ & Set of all types of space-time arcs \\
\hline$A^{T}$ & Set of space-time traveling arcs, $A^{T} \in A$ \\
\hline$A^{W}$ & Set of space-time waiting arcs, $A^{W} \in A$ \\
\hline$A^{A}$ & Set of space-time activity-performing arcs, $A^{A} \in A$ \\
\hline$A^{V}$ & Set of virtual traveling arcs, $A^{V} \in A$ \\
\hline$t, s, \tau$ & Indices of different time stamps, $t, s, \tau \in H$ \\
\hline$i, j$ & Indices of nodes, $i, j \in N$ \\
\hline$o$ & Index of origin nodes, $o \in P$ \\
\hline$a$ & Index of activity location nodes, $a \in P$ \\
\hline$(i, t),(j, s)$ & Indices of space-time vertexes, $(i, t),(j, s) \in Q$ \\
\hline$(i, j)$ & Index of physical link between adjacent nodes $i$ and $j,(i, j) \in E$ \\
\hline$(i, j, t, s)$ & $\begin{array}{l}\text { Index of space-time arcs indicating the actual movement at entering time } t \text { and } \\
\text { leaving time } s \text { on link }(i, j) \text {, arc }(i, j, t, s) \in A\end{array}$ \\
\hline$T(o, a)$ & $\begin{array}{l}\text { Total time budget for origin-activity location pair }(o, a) \text { in terms of number of } \\
\text { time intervals }\end{array}$ \\
\hline$T^{a}$ & Activity duration at activity location $a$ in terms of number of time intervals \\
\hline$T T(i, j, t)$ & Link travel time from node $i$ to node $j$ starting at time $t$ \\
\hline$c_{i, j, t, s}(o, a, \tau)$ & $\begin{array}{l}\text { Inaccessibility cost of arc }(i, j, t, s) \text { for origin-activity location pair }(o, a) \\
\text { departing at time } \tau(=1 \text {, for allvirtual traveling arcs; }=0 \text {, for all non-virtual arcs })\end{array}$ \\
\hline$g_{i, j, t, s}(o, a, \tau)$ & $\begin{array}{l}\text { Coefficient of space-time activity-performing arcs and virtual traveling arcs for the } \\
\text { activity at node } a \text { from node } o \text { departing at time } \tau(=1 \text {, for all }(i, j, t, s) \in \\
A^{A}, A^{V} ;=0 \text {, otherwise) }\end{array}$ \\
\hline$f_{i, j}$ & Construction cost of link $(i, j)$ \\
\hline$K$ & Total construction budget \\
\hline
\end{tabular}


Table 3 Decision variables used in mathematical formulations

\begin{tabular}{ll}
\hline Symbol & \multicolumn{1}{c}{ Definition } \\
\hline & $=1$, if a space-time traveling arc $(i, j, t, s)$ is used in the tour for origin-activity \\
& location pair $(o, a)$ departing at time $\tau ;$ \\
& $=0$, otherwise \\
& $=1$, if physical link $(i, j)$ is decided to be constructed in the transportation \\
& network; \\
& $=0$, otherwise \\
\end{tabular}


Table 4 Travel time data in hypothetic 3-node network

\begin{tabular}{lc}
\hline Link & Travel time \\
\hline$\left(o, a_{1}\right),\left(a_{1}, o\right)$ & 2 \\
\hline$\left(a_{1}, a_{2}\right),\left(a_{2}, a_{1}\right)$ & 3 \\
\hline$\left(o, a_{2}\right),\left(a_{2}, o\right)$ & 4 \\
\hline
\end{tabular}


Table 5 Strategies to increase accessibility

\begin{tabular}{|c|c|c|}
\hline Factors affected in Eq. (1) & Solution categories & Specific strategies \\
\hline \multirow{6}{*}{$\begin{array}{l}\text { Travel time } \\
\left(t^{\tau}(o, a) \text { and } t^{\tau^{\prime}}(a, o)\right)\end{array}$} & \multirow[b]{2}{*}{ Facility construction } & Capacity expansion \\
\hline & & $\begin{array}{l}\text { New road investment to connect major or new } \\
\text { activity locations }\end{array}$ \\
\hline & \multirow{2}{*}{$\begin{array}{l}\text { Providing other travel } \\
\text { options }\end{array}$} & $\begin{array}{l}\text { Public transit/ridesharing service improvement } \\
\text { (subways/ light rails/ buses/HOV/HOT lanes) }\end{array}$ \\
\hline & & Bicycle and pedestrian-friendly road design \\
\hline & \multirow{2}{*}{ Land use } & Activity location density adjustment \\
\hline & & Activity location distribution rationalization \\
\hline \multirow{3}{*}{$\begin{array}{l}\text { Departure time and } \\
\text { activity } \\
\text { participation } \\
\left(\tau \text { and } t_{a}^{m}\right)\end{array}$} & $\begin{array}{l}\text { Decreasing activity } \\
\text { duration }\end{array}$ & Activity duration decreasing \\
\hline & \multirow{2}{*}{ Avoiding congestion } & Shifting of departing time, demand spreading \\
\hline & & Shifting of routes, time-of-day pricing \\
\hline
\end{tabular}


Table 6 Travel time and construction cost data

\begin{tabular}{ccc}
\hline \multicolumn{1}{c}{ Link } & Travel time & Construction Cost \\
\hline$\left(o, a_{1}\right),\left(a_{1}, o\right)$ & 2 & 2 \\
\hline$\left(a_{1}, a_{2}\right),\left(a_{2}, a_{1}\right)$ & 3 & 3 \\
\hline$\left(o, a_{2}\right),\left(a_{2}, o\right)$ & 4 & 4 \\
\hline
\end{tabular}


Table 7 Impact of construction budget

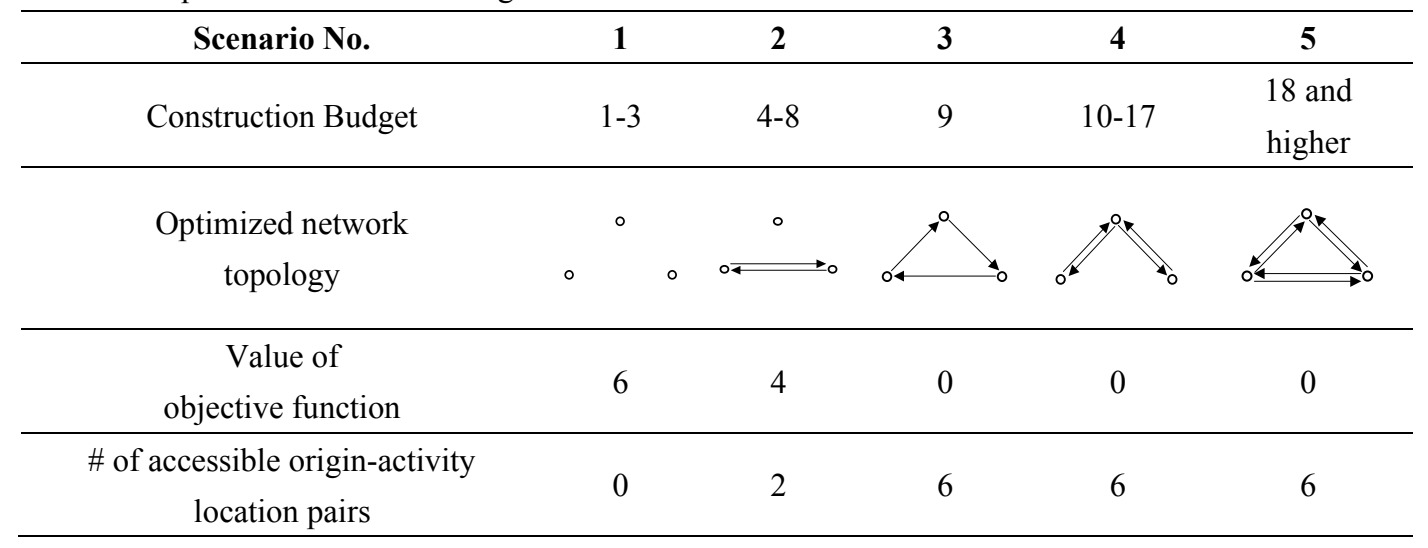


Table 8 Impact of travel time budget (under 10-unit construction budget)

\begin{tabular}{|c|c|c|c|c|c|c|}
\hline Scenario No. & 1 & & 2 & 3 & 4 & 5 \\
\hline Travel time budget & $2-5$ & & $6-7$ & $8-10$ & 11 & $\begin{array}{l}12 \text { and } \\
\text { higher }\end{array}$ \\
\hline $\begin{array}{l}\text { Optimized network } \\
\text { topology }\end{array}$ & $\circ$ & $\circ$ & $\begin{array}{c}0 \\
\stackrel{\circ}{\longrightarrow} 0\end{array}$ & 10 & 04 & \\
\hline Unused construction budget & 10 & & 6 & 0 & 1 & 0 \\
\hline $\begin{array}{c}\text { \# of accessible origin-activity } \\
\text { location pairs }\end{array}$ & 0 & & 2 & 4 & 6 & 6 \\
\hline
\end{tabular}


Table 9 Impact of link travel time (under 10-unit construction budget and 10-unit travel time budget)

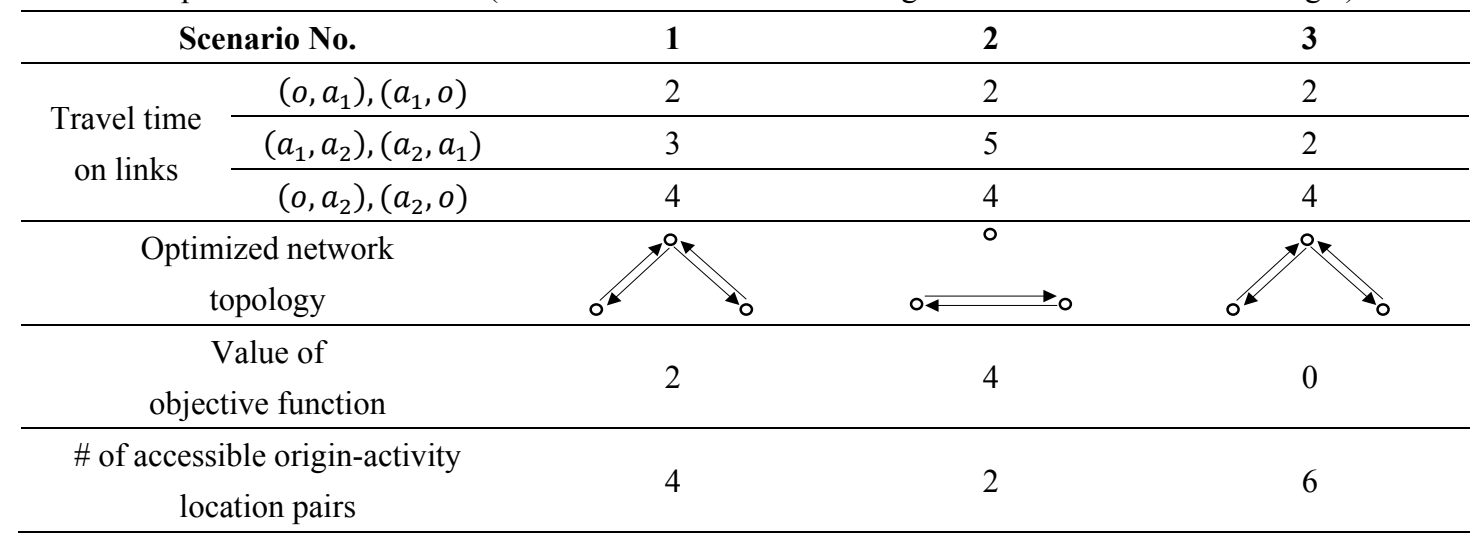


Table 10 Evolution of Lagrangian multipliers for all candidate links

\begin{tabular}{|c|c|c|c|c|c|c|c|c|c|c|}
\hline \multirow{2}{*}{$\#$} & \multirow{2}{*}{ step size } & \multicolumn{5}{|c|}{$\boldsymbol{\pi}_{i, j}$} & \multirow{2}{*}{$L_{X}$} & \multirow{2}{*}{$L_{y}$} & \multirow{2}{*}{ LB } & \multirow{2}{*}{$\begin{array}{c}\text { Gap } \\
\text { (UB-LB }\end{array}$} \\
\hline & & 1,5 & 2,5 & 5,6 & 6,3 & 6,4 & & & & \\
\hline 1 & -- & 0 & 0 & 0 & 0 & 0 & -- & -- & & \\
\hline 2 & 1 & 1 & 0 & 1 & 0 & 1 & 1 & -3 & -2 & 2 \\
\hline 3 & 0.6 & 0.4 & 0 & 0.4 & 0 & 0.4 & 1 & -1.2 & -0.2 & 0.2 \\
\hline 4 & 0.1 & 0.3 & 0 & 0.3 & 0 & 0.3 & 0.9 & -0.9 & 0 & 0 \\
\hline
\end{tabular}


Table 11 Computational statistics for Chicago sketch network

\begin{tabular}{lccccccccc}
\hline \multicolumn{1}{c}{ Iteration No. } & $\mathbf{1}$ & $\mathbf{2}$ & $\mathbf{3}$ & $\mathbf{4}$ & $\mathbf{5}$ & $\mathbf{1 0}$ & $\mathbf{2 0}$ & $\mathbf{3 0}$ & $\mathbf{4 0}$ \\
\hline Relative solution sap (\%) & 96.623 & 4.001 & 3.47 & 3.47 & 3.47 & 2.783 & 0.57 & 0.165 & 0.165 \\
\hline Cumulative CPU time (mm:ss) & $00: 40$ & $02: 26$ & $04: 10$ & $05: 53$ & $07: 37$ & $16: 16$ & $33: 31$ & $50: 47$ & $68: 04$ \\
\hline
\end{tabular}




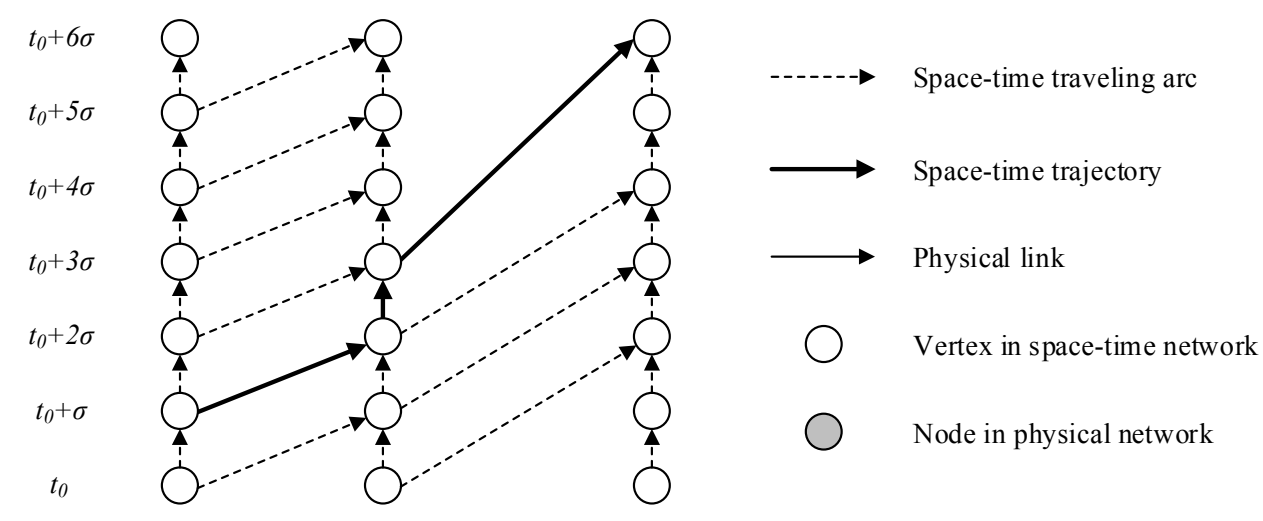

Physical network

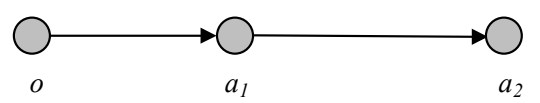

Fig 1. Illustration of physical network and space-time network (used in transportation network modeling literature) 


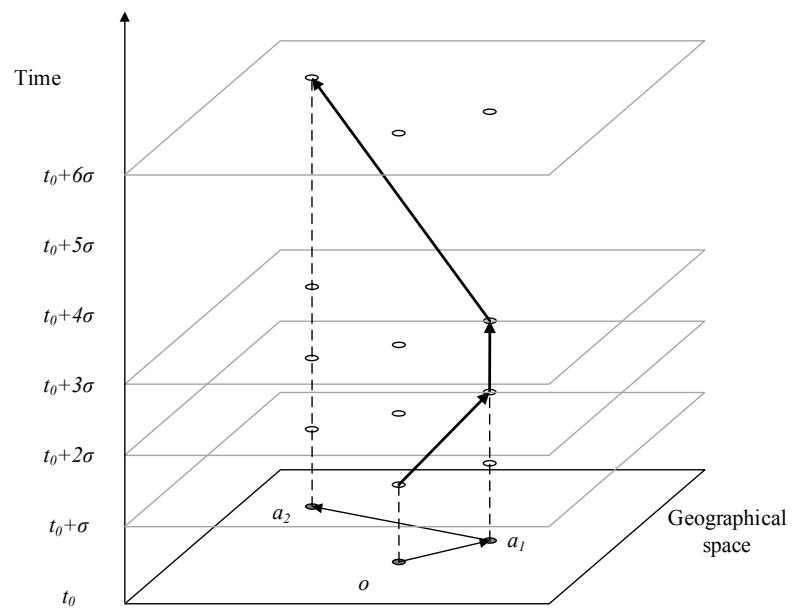

Fig 2. Illustration of a space-time path (adapted from Hägerstrand 1970) 


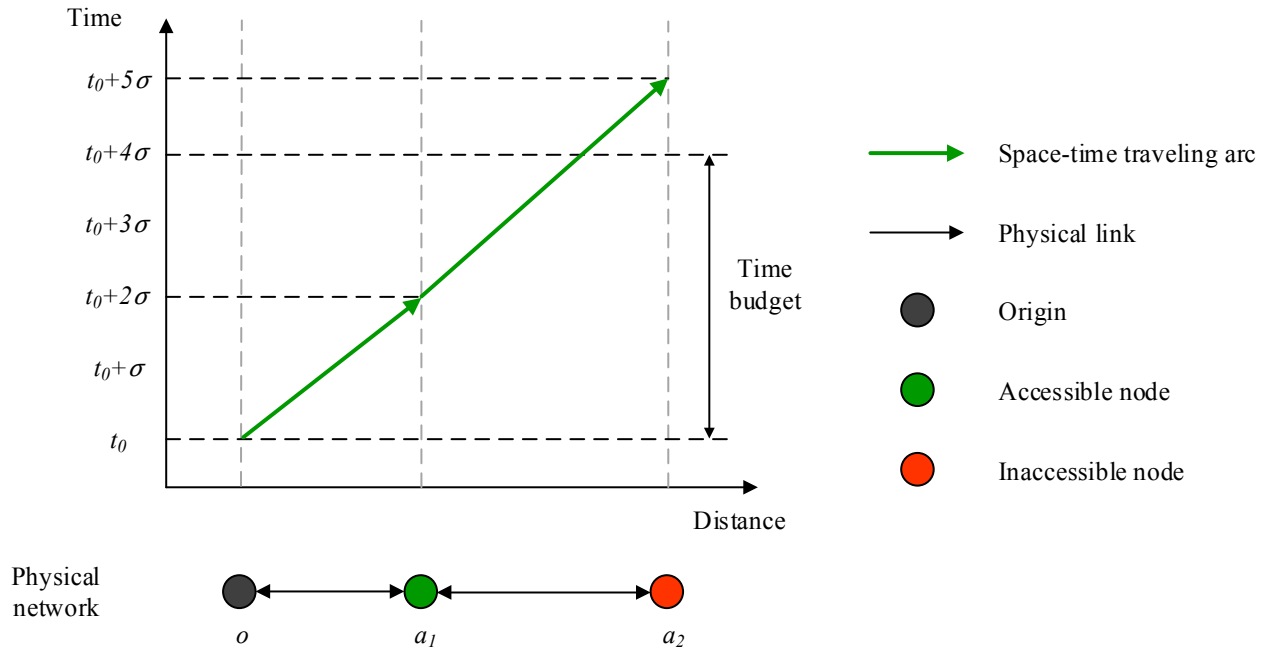

Fig 3. An illustration of accessibility concept from origin node to other nodes 


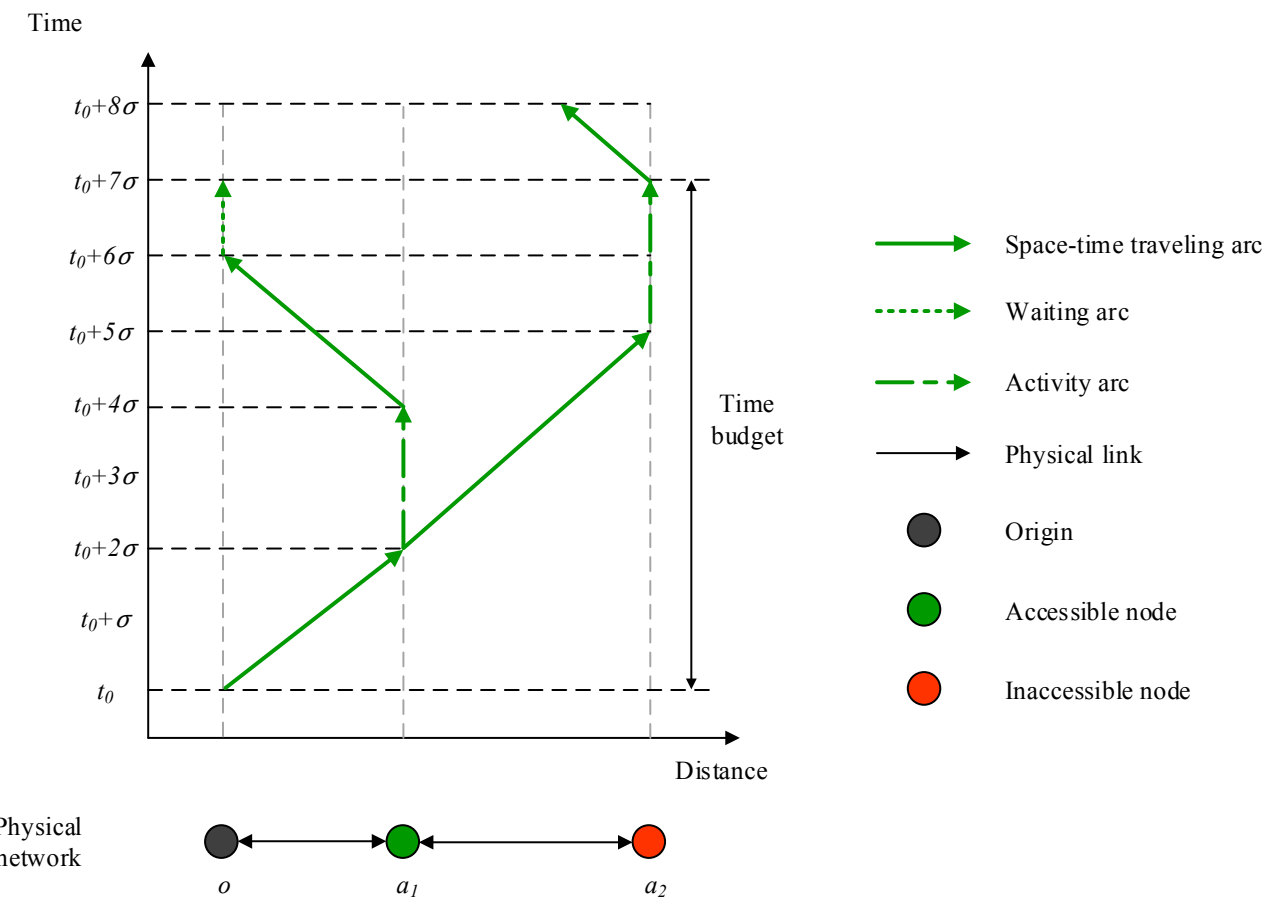

Fig 4. Accessible vs. inaccessible nodes for road users with traveling time constraints 


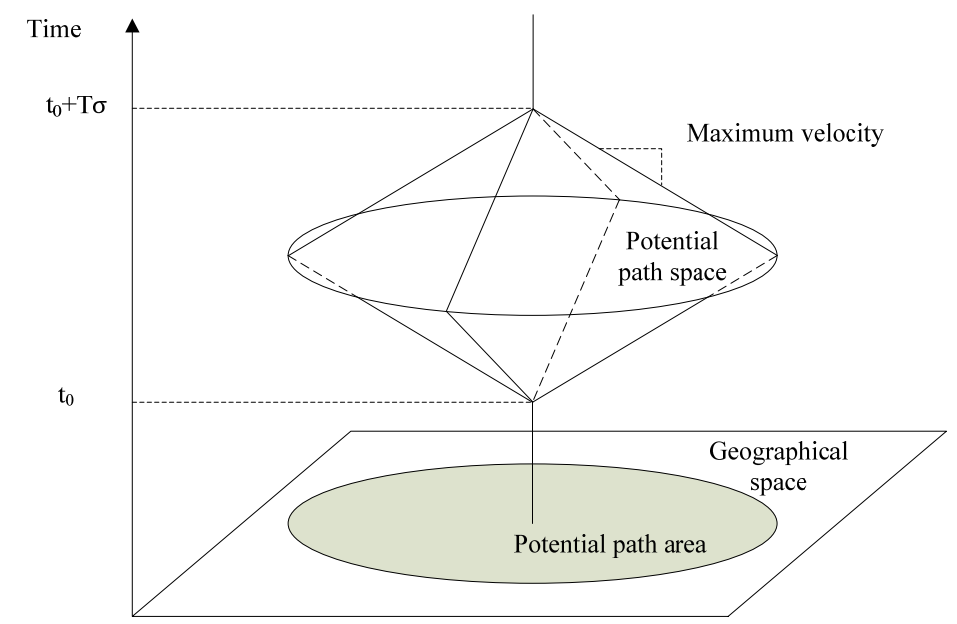

Fig 5. A simple space-time prism (Miller, 2005) 


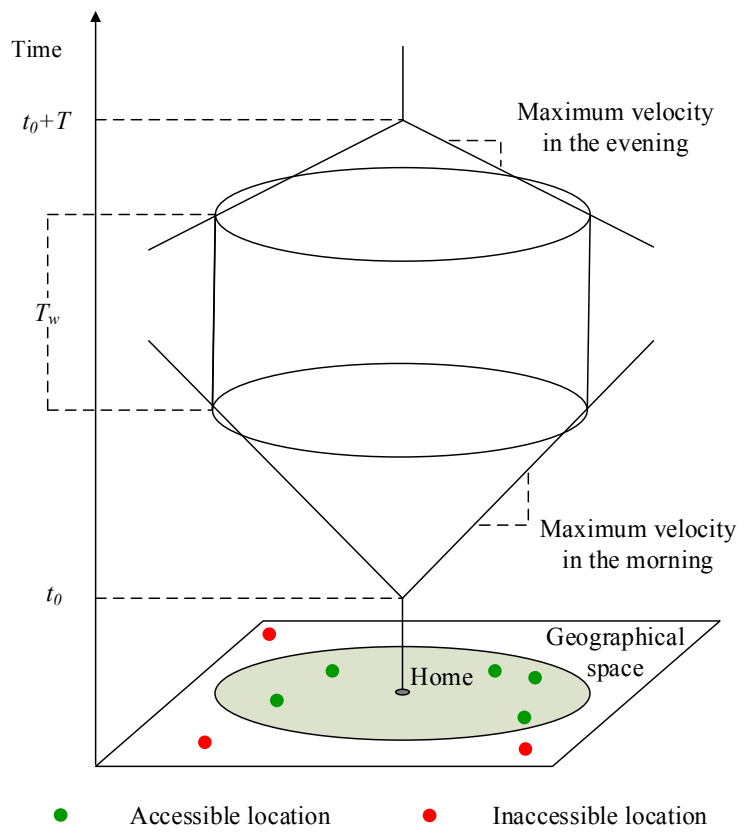

Fig 6.Mapping of accessible/ inaccessible locations with respect to potential activity area 


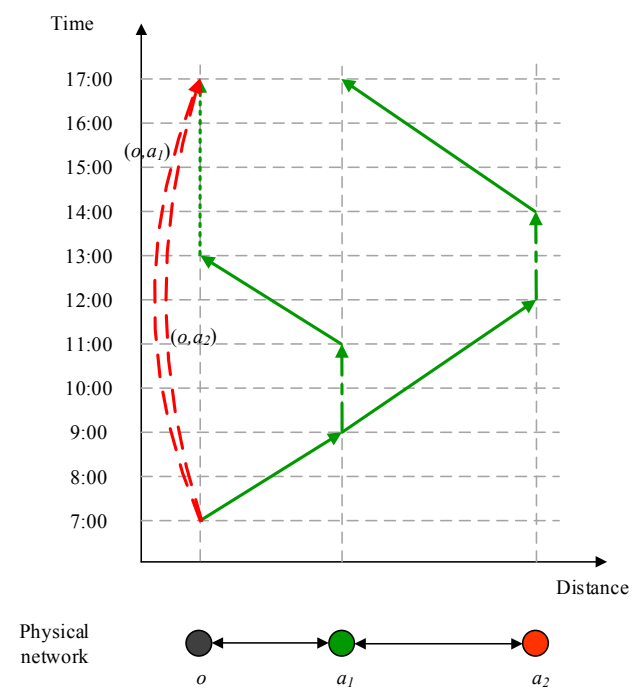

(a) Without link $\left(o, a_{2}\right)$

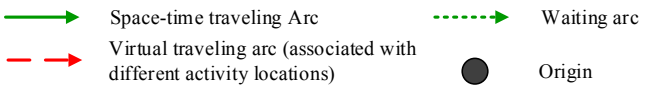

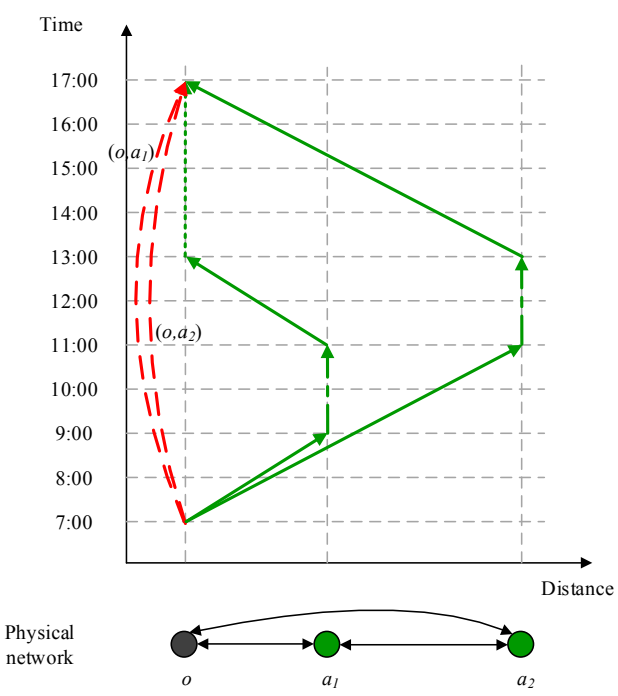

(b) With link $\left(o, a_{2}\right)$

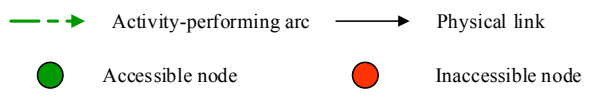

Fig 7. An illustration of adding a link in physical network 


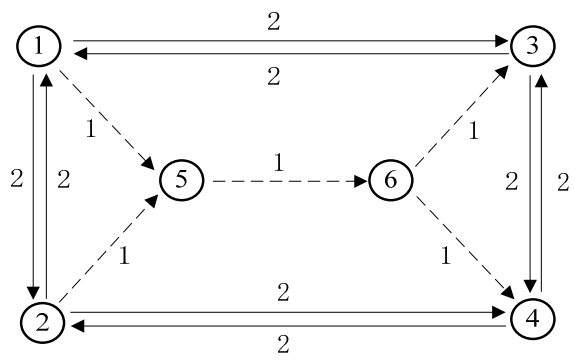

Fig 8. An illustration of adding links in physical network 


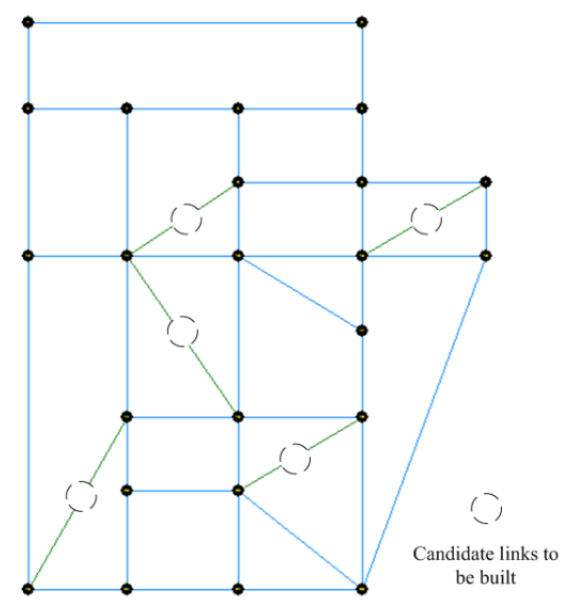

Fig 9. Simplified Sioux Falls network with 10 directional candidate links 


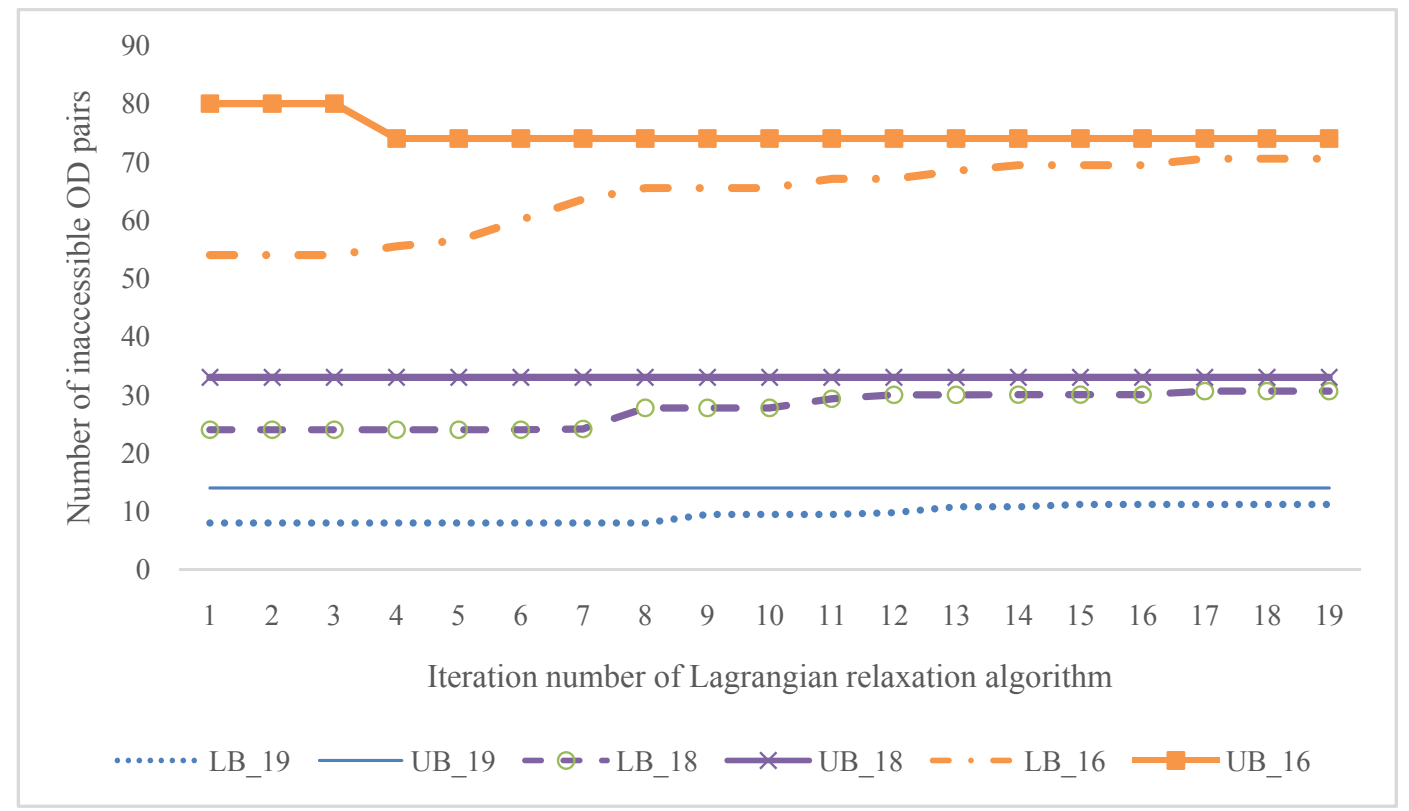

Fig 10. Evolution of $U B$ and $L B$ values for different travel time budgets (each time series is labeled as LB_TTB or UB_TTB for TTB $=19,18$, and 16 min) 


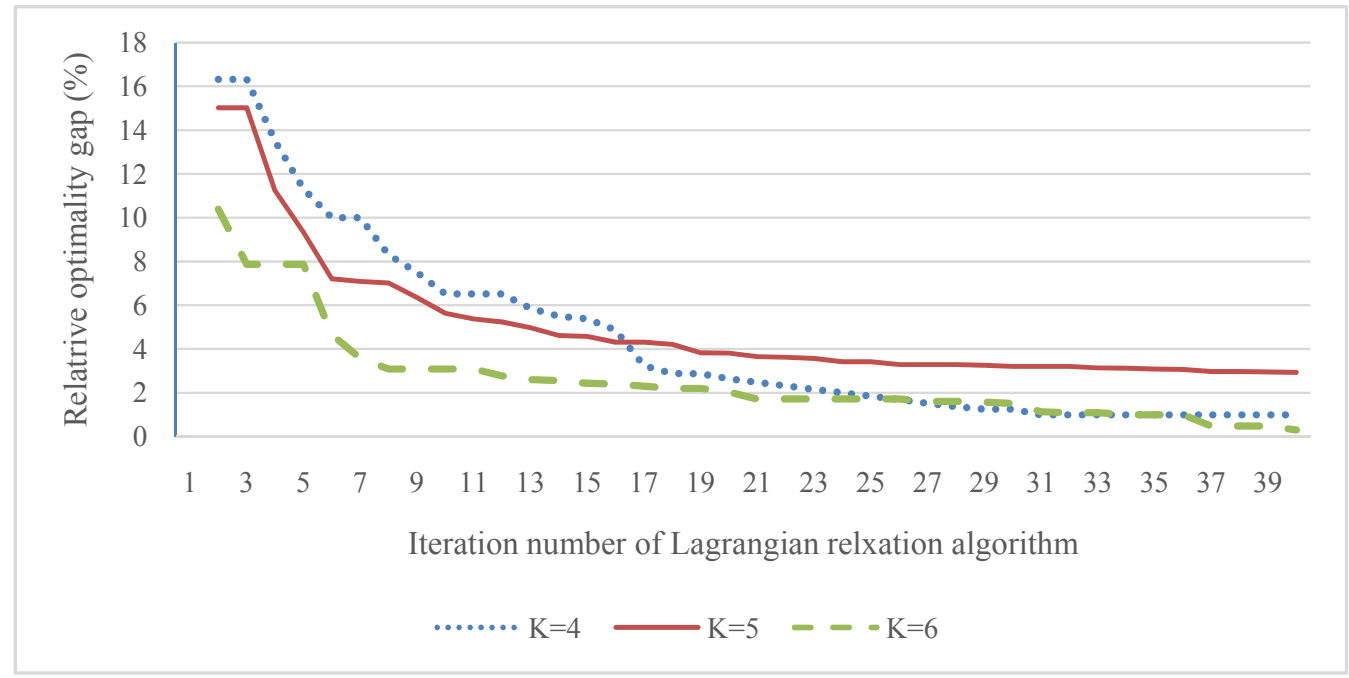

Fig 11. Evolution of relative optimality gap ((UB-LB)/UB) travel time budget $\mathrm{TTB}=12 \mathrm{~min}$, number of links to be selected $\mathrm{K}=4,5$ and 6 


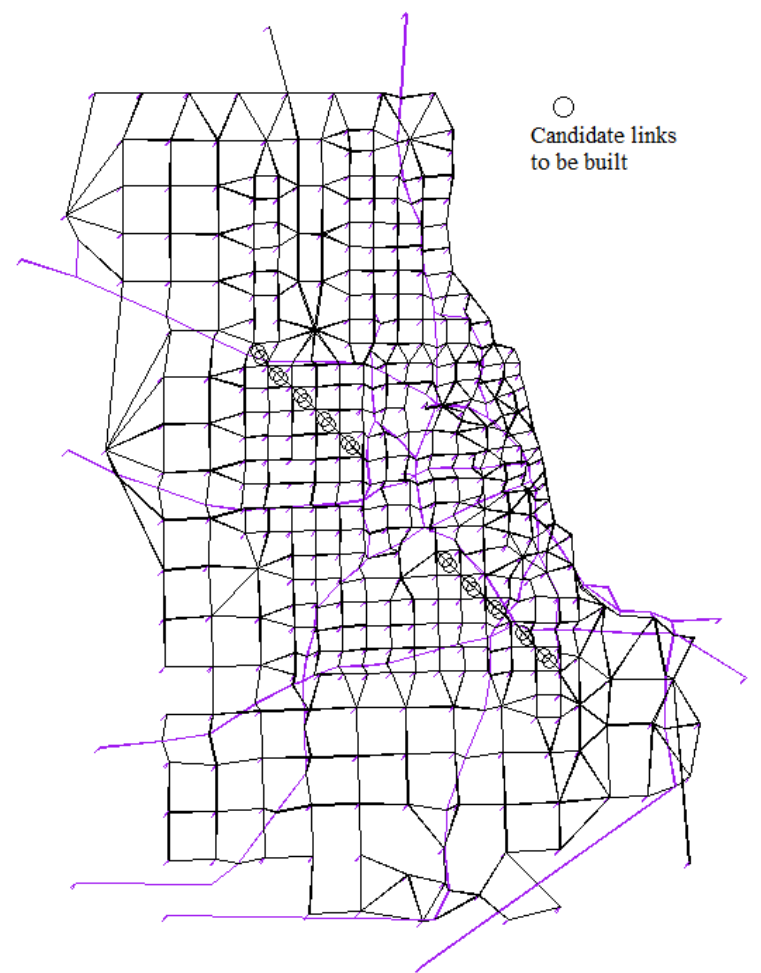

Fig 12.Chicago sketch network with 933 nodes, 2950 links, 387 zones and 20 (hypothetic) candidate links to be built 


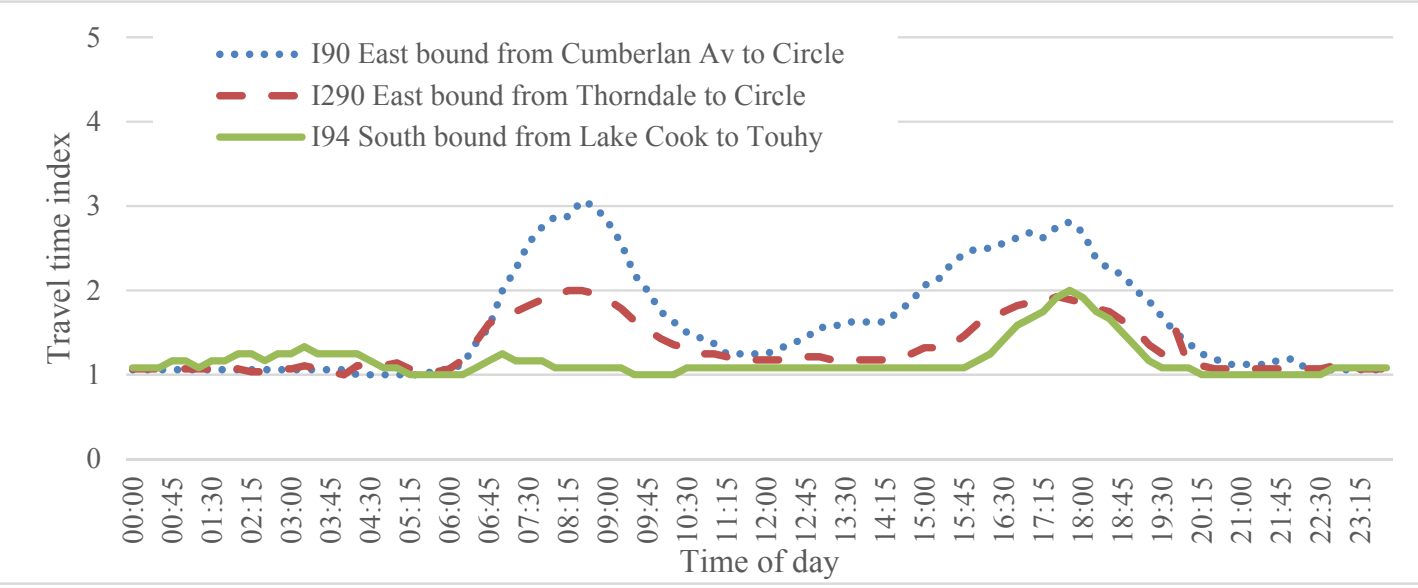

Fig 13. Sample corridor-level travel times across a day (travel time index $=$ travel time/free-flow travel time) 


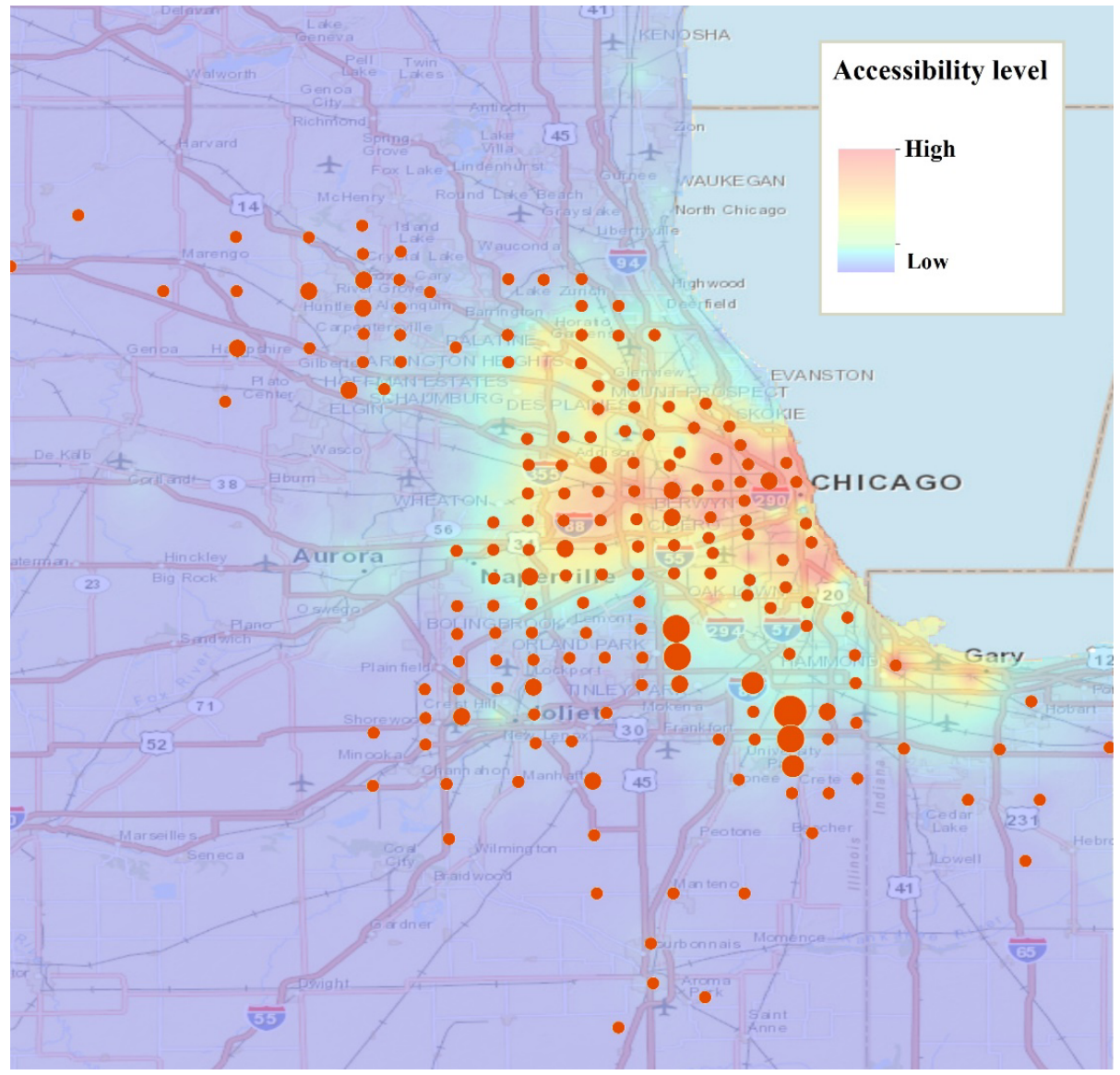

Fig 14. Accessibility heat map from the Chicago numerical testing results at 8:00 AM (the size of nodes reflects the additional number of activity locations accessible) 


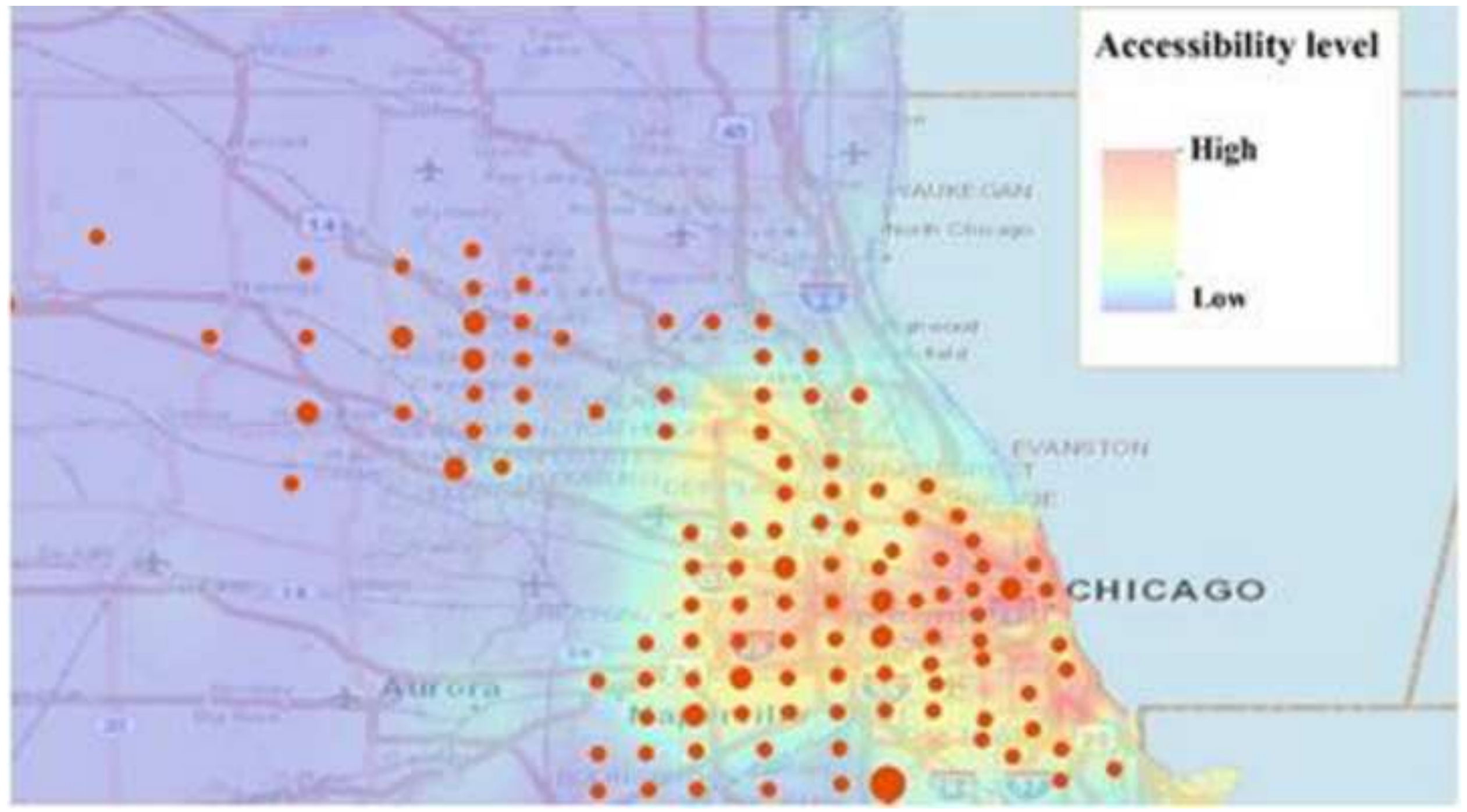

